\title{
On the Reachability of a Feedback Controlled Leontief-Type Singular Model Involving Scheduled Production, Recycling and Non-Renewable Resources
}

\author{
Manuel De la Sen ${ }^{1, *(D)}$, Asier Ibeas ${ }^{2}\left(\mathbb{D}\right.$ ) and Santiago Alonso-Quesada ${ }^{1}(\mathbb{D}$ \\ 1 Department of Electricity and Electronics, Faculty of Science and Technology, \\ Institute of Research and Development of Processes, Campus of Leioa, University of the Basque Country, \\ 48940 Leioa, Spain; santiago.alonso@ehu.eus \\ 2 Department of Telecommunications and Systems Engineering, Universitat Autònoma de Barcelona (UAB), \\ 08193 Barcelona, Spain; Asier.Ibeas@uab.cat \\ * Correspondence: manuel.delasen@ehu.eus
}

Citation: De la Sen, M.; Ibeas, A.; Alonso-Quesada, S. On the Reachability of a Feedback Controlled Leontief-Type Singular Model Involving Scheduled Production, Recycling and Non-Renewable Resources. Mathematics 2021, 9, 2175. https://doi.org/10.3390/math9172175

Academic Editor: Mihaela Paun

Received: 20 July 2021

Accepted: 2 September 2021

Published: 6 September 2021

Publisher's Note: MDPI stays neutral with regard to jurisdictional claims in published maps and institutional affiliations.

Copyright: (c) 2021 by the authors. Licensee MDPI, Basel, Switzerland. This article is an open access article distributed under the terms and conditions of the Creative Commons Attribution (CC BY) license (https:/ / creativecommons.org/licenses/by/ $4.0 /)$.

\begin{abstract}
This paper proposes and studies the reachability of a singular regular dynamic discrete Leontief-type economic model which includes production industries, recycling industries, and nonrenewable products in an integrated way. The designed prefixed final state to be reached, under discussed reachability conditions, is subject to necessary additional positivity-type constraints which depend on the initial conditions and the final time for the solution to match such a final prescribed state. It is assumed that the model may be driven by both the demand and an additional correcting control in order to achieve the final targeted state in finite time. Formal sufficiency-type conditions are established for the proposed singular Leontief model to be reachable under positive feedback, correcting controls designed for appropriate demand/supply regulation. Basically, the proposed regulation scheme allows fixing a prescribed final state of economic goods stock in finite time if the model is reachable.
\end{abstract}

Keywords: Leontief economic model; Ghosh model; controllability; equilibrium points; singular systems; stability; positive systems

\section{Introduction}

Leontief-type discrete economic models describe economic processes where several interrelated production sectors are involved [1,2]. The simpler models are static, but they can also be dynamic and described by either differential or difference equations. The most relevant parameters are the entries of a flow coefficient matrix, or technology matrix $A$, whose $(i, j)$ entry indicates the amount of product $i$ required from one sector to produce one unity of the sector $j$. There is also another involved matrix, $E$, the stock coefficient matrix which may be singular, and whose entry $e_{i j}$ indicates the amount of product $i$ which has to be in stock to produce one unit of product $j$ in the next sampling period. In this more general case, the model is said to be of a descriptor type. The singularity of the stock matrix is due to the fact that, in general, not any sector needs stock from other sectors to complete its production [2]. The typical driving force of this model is the demanded production level. Most general Leontief models can involve more parameters as well as additional correcting controls. The Leontief model can also be continuous and given by degenerated differential equations mixed with coupled algebraic equations, a version of the above-mentioned potential singularity of the stock coefficient matrix [3]. Leontief models have also been invoked for inter-fuel replacements between fossil fuels and the impact of sulfur-dioxide regulation of fossil fuel choices. See, for instance [4]. In [5], a standard dynamic Leontief model is extended by the incorporation of a balance equation of non-renewable resources whose stocks decrease by exploiting primary resources. The controllability of the proposed model, with the primary control being consumption, is 
analyzed. The evolution of a sub-state of recycling products is incorporated later on in [6]. The consideration of a continuous-time non-singular Leontief model as a control system is also considered in [7], where the control action is a function of unproductive consumption. A necessary and sufficient condition for a discrete singular Leontief model to be regular is discussed in [8]. In particular, it is proved in that paper that, if the singular system is regular, then the vector of capital stocks serves a state vector for the underlying dynamic system in such a way that the singularity of the stock matrix surprisingly decreases the dynamic order by making the system description simpler rather than more complex.

In [9], the positivity of the solutions of a non-singular dynamic price Leontief model is investigated based on some algebraic properties of their parameterizing matrices. The robust numerical solution of the optimal control of Leontief models is studied in [10] while a survey on optimal measurements theory is provided in [11], with some emphasis to the properties of the resolvent set of singular regular systems of usefulness for Leontief models. On the other hand, the formal expressions and some related relevant properties of the projection operators to deal with the Showalter Sidorov initial condition are addressed in [12]. Such initial condition replaces the standard Cauchy one in regular singular differential systems, such as some types of Leontief economic models. The controllability of linear descriptor systems, where the matrix $E$ is rectangular and full row rank, is discussed in detail in [13] by introducing an auxiliary system whose state is defined by the combination of the state components of the primary system related by such a matrix. The controllability of a regular Leontief model is also dealt with in [14], and some references therein, by addressing the concept of inoperability, basically, a measure of how willful attacks, accidental events, or natural causes may set off a chained set of impacts on other interconnected systems. Vulnerability in transportation and other complex networks is also discussed, for instance, in [15], by using a graph theoretical formalism. In [16], the so-called Ghosh model, which is basically supply-driven, so that each commodity is sold to each sector in fixed proportions, is discussed and compared to the Leontief model which is demand-driven. See also [17] and some references therein. On the other hand, some basic properties of a static so-called Armington-Leontief non-singular model, which includes the basic non-singular Leontief model, are formally discussed in [18]. The properties of positivity and productivity (i.e., for any non-negative vector of final demand, there is a non-negative output vector) are investigated.

It turns out that the positivity of the solutions under non-negative initial conditions and non-negative controls is an essential requirement for many systems because of their specific nature as it is, for instance, the case of those above-mentioned economic models and biological or epidemic models. See, for instance [19-24] and some of the references therein. This issue introduced collateral requirements in the discussion of the conditions of controllability, stability $[19,24]$, and model matching $[20,23]$, compared to the case of non-positive systems.

This paper describes a general proposed singular regular dynamic discrete Leontieftype economic model which considers coupled economic production industries, recycling industries, which used products, and non-renewable products. The model is subject to the usual driving demand plus a correcting control which is generated via feedback information of the economic production vector, recycling vector, and demand. The main objective is to study its reachability properties driven either by the demand vector or by the correcting control. It is well-known that reachability is a close concept to that of controllability of the state to the origin, but the main issue in the paper is to investigate the existence of a control which transfers any initial state to a prescribed final one in finite time. Due to the nature of the model (in particular, positive and non-singular regular), several specific difficulties have to be overcome, in particular, the achievement of the positivity of the solution sequence for any initial state under a positive control to be found which tracks a positive prefixed final state in finite time. Some constraints to be satisfied follow from the characteristics of the positive solution sequence and the positive control sequence which generates the solution. In particular, the final state is also subject to reinforced positivity 
constraints which depend on the initial conditions and the programmed final time for the solution to match the final prescribed state. It is assumed that the model is driven by both demand and a correcting control which can be, in turn, generated in the most general case by feedback information on the state variables and demand. The model is parameterized by the conventional input coefficients of production, the capital coefficients of production, the input coefficients of resources, showing the input of resources of industries which are required to produce a unit of product, the capital coefficients of recycling, used output production coefficients, recycling, as well as final consumption and the allocation matrix of output recycling coefficients related to the output of reused products.

Both the open-loop system (namely, the one described under a zero-correcting control) and the closed-loop system (namely, the one obtained under a nonzero correcting control) are dealt with. The conditions of non-negativity of the solution under a non-singularity particular constraint related to the re-usable goods substate are also formulated. Under such a simplification, the whole system might be still eventually singular. The reachability property of the above simplified model is also investigated by fixing a prefixed targeted production, via a demand monitoring on an appropriate set of samples. It is a crucial question to deal with the conditions of achievement of the reachability property under positive demands and positive prefixed targeted constrained production vectors.

In fact, a novelty in the paper is that it considers a very general singular discrete Leontief economic model with economic goods, reusable recycling goods, and non-renewable sources. The associated substates are coupled and integrated in the state of the general model. It turns out that the matrix of capital coefficients of production may be singular in many real cases, which motivates the setting of the model in a singular context. A mentioned case of singularity is related to the fact that not all economic goods produce relevant capital goods such as, for instance, agriculture. Therefore, the matrix of capital goods can have some zero rows which makes it singular. The proposed model incorporates a correcting feedback control which uses linear feedback from the measured state, and which is driven by the demand/supply error. The situation under a non-singular matrix of capital coefficients of production is also investigated as a particular case of the general mathematical framework.

A novelty in the paper is that it considers a very general singular discrete Leontief economic model with economic goods, reusable recycling goods, and non-renewable sources. The associated substates are coupled and integrated in the state of the general model. It turns out that the matrix of capital coefficients of production may be singular in many real cases, which motivates the setting of the model in a singular context. A mentioned case of singularity is related to the fact that not all economic goods produce relevant capital goods as, for instance, agriculture. Therefore, the matrix of capital goods can have some zero rows which makes it singular. The proposed model incorporates a correcting feedback control which uses linear feedback from the measured state, and which is driven by the demand/supply error. The situation under a non-singular matrix of capital coefficients of production is also investigated as a particular case of the general framework.

The main novelty of the article is the investigation of the reachability properties of the proposed model, in the sense that a prescribed value of the stock of economic goods can be prescribed in finite time and achieved by a certain demand. Such a demand is calculated as a function of the mentioned prescribed stock or by the above-mentioned correcting feedback control, which is driven by the demand/supply error and which is generated via feedback of the system state. The investigation of the mentioned reachability conditions is performed in the framework of singular discrete systems in the most general setting of the paper and, in particular, for the non-singular case as well. It has to be pointed out that the model singularity adds a relevant difficulty to the reachability analysis. On the other hand, numerical examples are performed to visualize the obtained results. The positivity of the model is also investigated since the relevant state variables have to be non-negative for all time and this feature should be compatible with the formulated reachability property. 
The paper is organized as follows. Section 2 studies both the open-loop system (i.e., that described under a zero-correcting control) and the closed-loop system (i.e., the one obtained under a nonzero correcting control). This section also formulates the conditions of non-negativity of the solution under a non-singularity particular constraint related on the reusable goods substate, with the whole system still being eventually singular. Section 3 relies on the reachability of the above simplified model, provided that an "ad hoc" reachability matrix is full rank, by fixing an appropriate set of successive non-negative demands which depends on each initial production vector condition and on each prefixed targeted production vector. To fix and develop the main ideas in the paper, the result is obtained under a technical assumption, which simplifies the exposition and the mathematical proof by keeping out the eventual singularity of the model. A collateral consequence of interest is that the set of demands used to achieve the targeting of the suited production vector after $n$ samples and the associated production vectors are all non-negative, so that the model is productive along any of such intervals of samples. Attention is paid to the positivity of the controls, which achieve the targeted reachability objective. Section 4 is devoted to the extension of the reachability study of Section 3 under the removal of the non-singularity constraint on re-usable goods. Section 5 discusses some numerical examples and, finally, a section of conclusions ends the paper.

\section{Notation}

$\boldsymbol{Z}_{0+}=\boldsymbol{Z}_{+} \cup\{0\}, \boldsymbol{R}_{0+}=\boldsymbol{R}_{+} \cup\{0\}$, The superscript "T" stands for the transpose of a real vector or matrix, $I_{p}$ denotes the $p$ identity matrix, $0_{p \times q} \in \boldsymbol{R}^{p \times q}$ denotes a zero matrix, $\bar{n}=\{1,2, \ldots, n\}$. Let $x, y \in \boldsymbol{R}^{n}$ and $X, Y \in \boldsymbol{R}^{n \times m}$ : Then $x \succ y$ (or $x-y \succ 0$, or $x-y \in \boldsymbol{R}_{0+}^{n}$ with $x \neq y$ ) denotes that $x \neq y$ and $x_{i} \geq y_{i} ; \forall i \in \bar{n}$ while $x \succ \succ y$ (or $x-y \succ \succ 0$, or $x-y \in \boldsymbol{R}_{+}^{n}$ ) denotes that $x_{i}>y_{i} ; \forall i \in \bar{n} ; x \succeq y$ denotes $x_{i} \geq y_{i} ; \forall i \in \bar{n}$; and $X \succ Y$ (or $X-Y \succ 0$, or $X-Y \in R_{0+}^{n \times m}$ with $X \neq Y$ ) denotes that $X \neq Y$ and $x_{i j} \geq y_{i j} ; \forall(i, j) \in \bar{n} \times \bar{m}$ while $X \succ \succ Y$ (or $X-Y \succ \succ 0$, or $X-Y \in R_{+}^{n \times m}$ ) denotes that $x_{i j}>y_{i j} ; \forall(i, j) \in \bar{n} \times \bar{m}$; $X \succeq Y$ denotes $x_{i j} \geq y_{i j} ; \forall(i, j) \in \bar{n} \times \bar{m} . x \succeq 0, X \succeq 0$ (i.e., they are non-negative), also denoted by $x \in \boldsymbol{R}_{0+}^{n}, X \in \boldsymbol{R}_{0+}^{n \times m}$, respectively, if they have no negative component or entry; $x \succ 0, X \succ 0$ (i.e., they are positive), also denoted by $x \in \boldsymbol{R}_{0+}^{n}, X \in \boldsymbol{R}_{0+}^{n \times m}$, respectively if they have no negative component or entry and at least one component or entry is positive; $x \succ \succ 0, X \succ \succ 0$ (i.e., they strictly positive), also denoted by $x \in \boldsymbol{R}_{+}^{n}, X \in \boldsymbol{R}_{+}^{n \times m}$ if all their components or entries are positive. Note that some literature defines strict positivity of vectors or matrices simply as positivity, and positivity simply as non-negativity.

\section{The Model and Its Solution Non-Negativity Properties under a Non-Singularity Constraint on the Re-Usable Goods Substate}

Consider the following discrete Leontief-type economic model:

$$
\begin{gathered}
x_{t}+P y_{t}=A_{x} x_{t}+B_{x}\left(x_{t+1}-x_{t}\right)+A_{y} y_{t}+B_{y}\left(y_{t+1}-y_{t}\right)+c_{t}+u_{t} \\
y_{t}=W_{x} x_{t}+W_{y} y_{t}+W_{c} c_{t} \\
R_{t+1}=R_{t}-D_{x} x_{t} \\
u_{t}=-K_{x} x_{t}-K_{y} y_{t}-K_{c} c_{t}
\end{gathered}
$$

where $t \in Z_{0+}$ is the sampling instant index, $n, m$, and $o$ are the numbers of economic industries, of recycling industries, each one recovering a used product, and of non-renewable products; $x_{t} \in \boldsymbol{R}^{n}, y_{t} \in \boldsymbol{R}^{m}$ and $R_{t} \in \boldsymbol{R}^{o}$ and $c_{t} \in \boldsymbol{R}_{0+}^{n}$ are, respectively, the stocks at the time instant $t$ of economic goods, those of used reusable recycling goods, those of non-renewable resources and product demand, subject to given initial conditions $x_{0} \in \boldsymbol{R}_{0+}^{n}, y_{0} \in \boldsymbol{R}_{0+}^{m}, R_{0} \in \boldsymbol{R}_{0+}^{o}$. The feedback linear correcting control $u_{t} \in \boldsymbol{R}^{n}$ is generated via (4) with the support of the product, recycling, and consumption control matrices $K_{x} \in \boldsymbol{R}^{n \times n}, K_{y} \in \boldsymbol{R}^{n \times m}$ and $K_{c} \in \boldsymbol{R}^{n \times n}$. Such a control adjusts the demand/supply error.

The matrices which parameterize (1)-(4) are: 
- $A_{x} \in \boldsymbol{R}^{n \times n}$ is the matrix of conventional input coefficients of production. Those coefficients show the inputs of goods of all the factories which are required to produce a unit of product,

- $A_{y} \in \boldsymbol{R}^{n \times m}$ is the matrix of input coefficients of recycling. Those coefficients show the inputs of goods of all the factories needed to recycle a unit of used product,

- $B_{x} \in \boldsymbol{R}^{n \times n}$ is the matrix of capital coefficients of production,

- $D_{x} \in \boldsymbol{R}^{o \times n}$ is the matrix of the input coefficients of resources showing the input of resources of industries which are required to produce a unit of product,

- $\quad B_{y} \in \boldsymbol{R}^{n \times m}$ is the matrix of capital coefficients of recycling,

- $\quad W_{x} \in \boldsymbol{R}^{m \times n}, W_{y} \in \boldsymbol{R}^{m \times m}$ and $W_{c} \in \boldsymbol{R}^{m \times n}$ are the matrices of used-output coefficients of production, recycling, and final consumption, respectively, and

- $\quad P \in \boldsymbol{R}^{n \times m}$ is the allocation matrix of output coefficients of recycling which shows the output of reused products produced by the recycling sectors in such a way that $x_{t}+P y_{t}$ are the new and remanufactured products which are used for production $A_{x} x_{t}$ and for recycling $A_{y} y_{t}$, respectively, for one year capital accumulations $B_{x}\left(x_{t+1}-x_{t}\right)$ and $B_{y}\left(y_{t+1}-y_{t}\right)$ in the production and the recycling sectors, respectively.

- The matrices $B_{x}$ and $B_{y}$ together with eventual zero block matrices and identity matrices are then re-arranged in an eventually singular matrix $E$ for a compact description of the singular Leontief model.

- A built compacted matrix containing the control gains $K_{x}, K_{y}$ for detailed feedback analysis will be denoted with $K$.

- The remaining relevant matrices of coefficients of production and input and output coefficients and the control gain $K_{c}$ will be grouped in extended matrices of openloop (or control-free) and closed-loop (including feedback information) dynamics, respectively, $A^{0}$ and $A$, or in a control matrix $B$.

Remark 1. (demand/supply error driven by the incremental feedback control). The controlled system under the demand and correcting control admits a nice interpretation in terms of demand and regulated supply issues. In this sense, the control action consists of modifying the supply according to the demand so as to correct the model behavior. We focus the next related discussion on how the substate $x_{t}$ of economic goods is regulated via feedback information based on demand and supply. Define the supply at the time instant $s_{t}$ and the demand/supply error becomes $e_{t}=c_{t}-s_{t}$. It is now seen through simple manipulations with (1)-(4) that the supply in the market is partially driven via feedback by the correcting control $u_{t}$ which is, in fact, the minus demand/supply error. Looking at (1) and (4), we might rewrite equivalently (1) as:

$$
B_{x} x_{t+1}=B_{x} x_{t}+\left(I_{n}-A_{x}\right) x_{t}-s_{t}=B_{x} x_{t}+\left(I_{n}-A_{x}\right) x_{t}+e_{t}-c_{t}
$$

where

$$
\begin{aligned}
& s_{t}=-K_{x} x_{t}+B_{y} y_{t+1}+\left(A_{y}-P-B_{y}-K_{y}\right) y_{t}+\left(I_{n}-K_{c}\right) c_{t} \\
& =B_{y}\left(y_{t+1}-y_{t}\right)+\left(A_{y}-P\right) y_{t}+c_{t}+u_{t} \\
& e_{t}=c_{t}-s_{t}=K_{x} x_{t}-B_{y}\left(y_{t+1}-y_{t}\right)+\left(P+K_{y}-A_{y}\right) y_{t}+K_{c} c_{t} \\
& =-B_{y}\left(y_{t+1}-y_{t}\right)+\left(P-A_{y}\right) y_{t}-u_{t} \\
& U_{t}=s_{t}-c_{t}-B_{y}\left(y_{t+1}-y_{t}\right)+\left(P-A_{y}\right) y_{t}
\end{aligned}
$$

so that it is easily interpreted from the above relations that the supply and the demand/supply error are correspondingly regulated with the feedback correcting control $u_{t}$ which uses feedback information. Note that the corresponding open-loop (i.e., correcting control-free, that is, $u_{t} \equiv 0$ ) versions of the above sequences are obtained by zeroing the three feedback gain matrices, leading to:

$$
\begin{gathered}
s_{t}^{0}=B_{y}\left(y_{t+1}-y_{t}\right)+\left(A_{y}-P\right) y_{t}+c_{t} \\
e_{t}^{0}=c_{t}-s_{t}^{0}=-B_{y}\left(y_{t+1}-y_{t}\right)+\left(P-A_{y}\right) y_{t} \\
u_{t}^{0}=s_{t}^{0}-c_{t}-B_{y}\left(y_{t+1}-y_{t}\right)+\left(P-A_{y}\right) y_{t}=s_{t}^{0}+e_{t}^{0}-c_{t}=0
\end{gathered}
$$


so that there is no correcting control in this case, as expected.

Now, assume, in particular, that there is no re-usable substate considered in the model, i.e., $y_{t} \equiv 0$, then $u_{t}=s_{t}-c_{t}=-e_{t} ; u_{t}^{0}=s_{t}^{0}-c_{t}=-e_{t}^{0}$. Note from the above relations that the supply/demand error $s_{t}-c_{t}$ is regulated by the gains $K_{x}, K_{y}$, and $K_{c}$ of the auxiliary control $u_{t}=-e_{t}$, which take feedback information of the economic and recycling goods state $y_{t}$ and of the demand $c_{t}$.

Roughly speaking, the production system consists of economic and recycling industries and of non-renewable products. The state of the whole economic system contains the coupled substates of the above objects. The stock of economic goods should try to satisfy the demands of products. It turns out that the assumption of non-singularity of the matrix of capital coefficients of production $B_{x}$ in (1) is not usually justified. Note, for instance, that its $(i, k)$ entry is the stock amount of commodity $i$, as capital good, that the sector $k$ has to manage per unity of production. It turns out that not all sectors produce relevant capital goods, for instance, agriculture. Therefore, it is sometimes convenient to state economic models where the above matrix has some zero rows, so that it becomes singular. This is a relevant motivation for the idea of stating singular Leontief models, as proposed in the main body of this paper.

The matrix of capital recycling coefficients $B_{y}$ can also be singular in the most general case. In addition, when the model (1)-(4) is rewritten in a compact way by grouping its three integrating substates, they appear as new zero block matrices in the leading resulting matrix of the compacted discrete dynamical systems as it is seen immediately after in Equations (5)-(12). This fact reinforces the usefulness of describing the system in the framework of singular discrete dynamic systems. Therefore, the paper addresses the model reachability conditions in both the non-singular and singular situations in order to be able to satisfy some prefixed substate of economic goods in a given finite discrete time. It is investigated how to achieve the above objective through either monitoring the demand level or through designing the correcting control by the design of its gain matrices $K_{x}, K_{y}$, and $K_{c}$. This second mechanism becomes more useful in practice for its application in a market-free economy since is not interventionist on monitoring the demand, but it designs the control matrices to regulate the demand/supply error so as to achieve the prefixed reachability objective of suited stock of economic goods (see also Remark 1).

The closed-loop, or controlled, model (1)-(4) may be rewritten, for later description convenience, more compactly as follows by defining the extended state-vector $z_{t}=\left(x_{t}^{T}, y_{t}^{T}, R_{t}^{T}\right)^{T} \in \boldsymbol{R}^{n+m+o}$ :

$$
E z_{t+1}=A z_{t}+B c_{t}
$$

where:

$$
E=\left[\begin{array}{ccc}
B_{x} & B_{y} & 0_{n \times o} \\
0_{m \times n} & 0_{m \times m} & 0_{m \times o} \\
0_{o \times n} & 0_{o \times m} & I_{o}
\end{array}\right] ; A=\left[\begin{array}{ccc}
C_{x} & D_{y} & 0_{n \times o} \\
W_{x} & W_{y}-I_{m} & 0_{m \times o} \\
-D_{x} & 0_{o \times m} & I_{o}
\end{array}\right] ; B=\left[\begin{array}{c}
E_{c} \\
W_{c} \\
0_{o \times n}
\end{array}\right]
$$

$C_{x} \in \boldsymbol{R}^{n \times n}, D_{y} \in \boldsymbol{R}^{n \times m}$ and $E_{c} \in \boldsymbol{R}^{n \times n}$ are defined by:

$$
\begin{gathered}
C_{x}=I_{n}+B_{x}-A_{x}+K_{x} \\
D_{y}=B_{y}+P-A_{y}+K_{y} \\
E_{c}=K_{c}-I_{n}
\end{gathered}
$$

The open-loop (that is, uncontrolled) model is obtained, subject to identically zero correcting control $u_{t} \equiv 0$, achieved by zeroing the three control matrices, and results to be:

$$
E z_{t+1}=A^{0} z_{t}+B^{0} c_{t}
$$


where:

$$
A^{0}=\left[\begin{array}{ccc}
C_{x}^{0} & D_{y}^{0} & 0_{n \times o} \\
W_{x} & W_{y}-I_{m} & 0_{m \times o} \\
-D_{x} & 0_{o \times m} & I_{o}
\end{array}\right] ; B^{0}=\left[\begin{array}{c}
E_{c}^{0} \\
W_{c} \\
0_{o \times n}
\end{array}\right]
$$

with

$$
\left.\left.\left.C_{x}^{0}=C_{x}\right]_{K_{x}=0}=I_{n}+B_{x}-A_{x} ; D_{y}^{0}=D_{y}\right]_{K_{y}=0}=B_{y}+P-A_{y} ; E_{c}^{0}=E_{c}\right]_{K_{c}=0}=-I_{n}
$$

Remark 2. (existence of solution of re-usable products). Note that the solutions $\left(x_{t}^{T}, y_{t}^{T}, R_{t}^{T}\right)^{T}$ of (5) are in $\operatorname{Ker}\left[W_{x}, W_{y}-I_{m}, W_{c}\right]$. In particular, note also that for each given solution productiondemand pairs $\left(x_{t}, c_{t}\right)$, a set of solutions of re-usable products $y_{t}$ to the equation $\left(I_{m}-W_{y}\right) y_{t}=$ $W_{x} x_{t}+W_{c} c_{t}$ exists if and only if $\operatorname{rank}\left[I_{m}-W_{y}\right]=\operatorname{rank}\left[I_{m}-W_{y}, W_{x} x_{t}+W_{c} c_{t}\right]$ and such a set which includes all the solutions, even the non-positive ones, is given by $y_{t}(z)=\left(I_{m}-W_{y}\right)^{\dagger}\left(W_{x} x_{t}+W_{c} c_{t}-\left(I_{m}-W_{y}\right) z\right)+z$ for any parameterizing vector $z \in \boldsymbol{R}^{n}$, where $\left(I_{m}-W_{y}\right)^{\dagger}$ is the Moore-Penrose pseudoinverse of $\left(I_{m}-W_{y}\right)$. If $\left(I_{m}-W_{y}\right)$ is full row rank then $y_{t}=\left(I_{m}-W_{y}\right)^{-1}\left(W_{x} x_{t}+W_{c} c_{t}\right)$ is the unique solution which is positive if either $\left(I_{m}-W_{y}\right)$ is non-singular positive monomial, that is if either $W_{y}$ is diagonal with entries less than one or if $\left(I_{m}-W_{y}\right)$ is a non-singular $M$-matrix (which holds if and only if all its principal minors are positive) since $W_{x} \succ 0, W_{y} \succ 0$ and $W_{c} \succ 0$.

Note that (5) and (8) hold irrespective of $\left(I_{m}-W_{y}\right)$ being non-singular or not. The following result relies on the simplified model that holds directly when $\left(I_{m}-W_{y}\right)$ is nonsingular so that $y_{t}(z)=y_{t}(0)$ is a unique solution. Note that the whole model might still be singular, since $E$ can be still singular in spite of $\left(I_{m}-W_{y}\right)$ being non-singular.

Proposition 1. If $\left(I_{m}-W_{y}\right)^{-1}$ exists, then

$$
\bar{B}_{x} x_{t+1}=\bar{C}_{x} x_{t}+\bar{E}_{c} c_{t}-B_{y}\left(I_{m}-W_{y}\right)^{-1} W_{c} c_{t+1}
$$

which becomes to be in the uncontrolled case, i.e., for $u_{t} \equiv 0$,

$$
\bar{B}_{x} x_{t+1}=\bar{C}_{x}^{0} x_{t}+\bar{E}_{c}^{0} c_{t}-B_{y}\left(I_{m}-W_{y}\right)^{-1} W_{c} c_{t+1}
$$

where

$$
\begin{gathered}
\bar{B}_{x}=B_{x}+B_{y}\left(I_{m}-W_{y}\right)^{-1} W_{x} \\
\bar{C}_{x}=C_{x}+D_{y}\left(I_{m}-W_{y}\right)^{-1} W_{x} ; \bar{C}_{x}^{0}=C_{x}^{0}+D_{y}^{0}\left(I_{m}-W_{y}\right)^{-1} W_{x} \\
\bar{E}_{c}=E_{c}+D_{y}\left(I_{m}-W_{y}\right)^{-1} W_{c} ; \bar{E}_{c}^{0}=E_{c}^{0}+D_{y}^{0}\left(I_{m}-W_{y}\right)^{-1} W_{c}
\end{gathered}
$$

Proof. It follows from (2) that

$$
y_{t}=\left(I_{m}-W_{y}\right)^{-1}\left(W_{x} x_{t}+W_{c} c_{t}\right)
$$

which replaced in (1), while using (7)-(9) and (15)-(17), leads to (13) which is a re-ordering of terms of the following identity: 


$$
\begin{aligned}
& B_{x} x_{t+1}=\left(I_{n}+B_{x}-A_{x}+K_{x}\right) x_{t}+\left(B_{y}+P-A_{y}+K_{y}\right) y_{t}-B_{y} y_{t+1}+\left(K_{c}-I_{n}\right) c_{t} \\
& =\left[I_{n}+B_{x}-A_{x}+K_{x}+\left(B_{y}+P-A_{y}+K_{y}\right)\left(I_{m}-W_{y}\right)^{-1} W_{x}\right] x_{t} \\
& +\left[K_{c}-I_{n}+\left(B_{y}+P-A_{y}+K_{y}\right)\left(I_{m}-W_{y}\right)^{-1} W_{c}\right] c_{t}-B_{y} y_{t+1} \\
& =\left[I_{n}+B_{x}-A_{x}+K_{x}+\left(B_{y}+P-A_{y}+K_{y}\right)\left(I_{m}-W_{y}\right)^{-1} W_{x}\right] x_{t} \\
& +\left[K_{c}-I_{n}+\left(B_{y}+P-A_{y}+K_{y}\right)\left(I_{m}-W_{y}\right)^{-1} W_{c}\right] c_{t}-B_{y}\left(I_{m}-W_{y}\right)^{-1}\left(W_{x} x_{t+1}+W_{c} c_{t+1}\right) \\
& =\left(C_{x}+D_{y}\left(I_{m}-W_{y}\right)^{-1} W_{x}\right) x_{t} \\
& +\left(K_{c}-I_{n}+D_{y}\left(I_{m}-W_{y}\right)^{-1} W_{c}\right) c_{t}-B_{y}\left(I_{m}-W_{y}\right)^{-1} W_{x} x_{t+1}-B_{y}\left(I_{m}-W_{y}\right)^{-1} W_{c} c_{t+1}
\end{aligned}
$$

Equation (14) is obtained with the use of (12).

Proposition 1 leads to the following equilibrium results:

Proposition 2. (existence and uniqueness of the equilibrium point). If $\left(I_{m}-W_{y}\right)^{-1}$ exists then the equilibrium points, if any, satisfy the relationship:

$$
\left(\bar{B}_{x}-\bar{C}_{x}\right) x=\left(\bar{E}_{c}-B_{y}\left(I_{m}-W_{y}\right)^{-1} W_{c}\right) c
$$

and

(a) the equilibrium point $x$ is unique for any given constant demand $c$ if, and only if, $\bar{B}_{x}-\bar{C}_{x}$ is non-singular, while $x \succ 0$ if $\left(\bar{B}_{x}-\bar{C}_{x}\right)$ is an $M$-matrix, $\bar{E}_{c}-B_{y}\left(I_{m}-W_{y}\right)^{-1} W_{c} \succ 0$ and $c \succ 0$ which guarantee a well-posed equilibrium problem.

(b) the equilibrium point $x$ is non-unique for any given $c$ if and only if $\bar{B}_{x}-\bar{C}_{x}$ is singular and $\operatorname{rank}\left(\bar{B}_{x}-\bar{C}_{x}\right)=\operatorname{rank}\left(\bar{B}_{x}-\bar{C}_{x}, \bar{E}_{c}-B_{y}\left(I_{m}-W_{y}\right)^{-1} W_{c}\right)$ while $x \succ 0$ if $\left(\bar{B}_{x}-\bar{C}_{x}\right)$ is an M-matrix, $\bar{E}_{c}-B_{y}\left(I_{m}-W_{y}\right)^{-1} W_{c} \succ 0$ and $c \succ 0$, which guarantee a well-posed equilibrium problem.

(c) the condition (20) comes to be in the uncontrolled situation:

$$
\left(\bar{B}_{x}-\bar{C}_{x}^{0}\right) x^{0}=\left(\bar{E}_{c}^{0}-B_{y}\left(I_{m}-W_{y}\right)^{-1} W_{c}\right) c^{0}
$$

The conditions of existence, uniqueness, and positivity of the equilibrium consumption are similar to the above ones by replacing $\bar{C}_{x} \rightarrow \bar{C}_{x}^{0}, \bar{E}_{c} \rightarrow \bar{E}_{c}^{0}, c \rightarrow c^{0}$.

Proof. Equations (20) and (21) follow from (13) and (14) with $x_{t}=x_{t+1}=x=x^{0}$ and $c_{t}=c_{t+1}=c^{0} ; \forall t \in \mathbf{Z}_{0+}$. The conditions of existence, uniqueness, and positivity follow from the Rouché-Capelli theorem for compatible systems and from the fact that the inverse of an $M$-matrix is positive.

The properties of positive and singular matrices or systems are discussed in [25-31]. Some useful techniques for factorization of $M$-matrices are found, for instance, in [32-34] and some of the references therein.

\section{Reachability Properties under the Non-Singularity Constraint on the Re-Usable Goods Substate}

In order for the model to be well-posed, the subsequent assumption is made towards the solution positivity:

Assumption 1. $B_{x} \succ 0, B_{y} \succ 0, A_{x} \succ 0, P \succ 0, W_{x} \succ 0, W_{y} \succ 0$ and $W_{\mathcal{c}} \succ 0$.

The next result is a reachability result of a prefixed non-negative production vector in $(n+1)$ samples starting with any non-negative initial condition fixed at any arbitrary sampling instant, which satisfies a non-negative error related to the non-negative initial 
production vector. The objective is achievable by an appropriate set of successive nonnegative demands which depends on each initial production vector condition and on each prefixed targeted production vector provided that an "ad hoc" reachability matrix is non-singular. To fix ideas, the result is obtained under Assumption 1 which simplifies the exposition and the mathematical proof by keeping out the eventual singularity of the model. A collateral consequence of interest is that the set of demands used to achieve the targeting of the suited production vector after $n$ samples and the associated production vectors are all non-negative, so that the model is productive along any such intervals of samples.

Theorem 1. (reachability of the vector of economic goods from the demand). Consider the uncontrolled model (1)-(3) and assume that

(1) Assumption 1 holds,

(2) $\bar{C}_{x}^{0} \succ 0, \bar{E}_{c}^{0}-\bar{C}_{x}^{0} \bar{B}_{x}^{-1} B_{y}\left(I_{m}-W_{y}\right)^{-1} W_{c} \succ 0$,

(3) Either $I_{m}-W_{y} \succ 0$ and monomial (that is, $W_{y}$ is diagonal with positive diagonal entries smaller than one or $I_{m}-W_{y}$ is a non-singular M-matrix),

(4) Either $\bar{B}_{x} \succ 0$ is monomial or it is a non-singular M-matrix,

(5) $R_{c}$ has only a nonzero entry per row and $\operatorname{rank}\left(R_{c}\right)=n$, where

$$
R_{c}=\left[B_{1},\left(\bar{B}_{x}^{-1} \bar{C}_{x}^{0}\right) B_{1}, \cdots,\left(\bar{B}_{x}^{-1} \bar{C}_{x}^{0}\right)^{n-2} B_{1},\left(\bar{B}_{x}^{-1} \bar{C}_{x}^{0}\right)^{n-1} B_{2}\right] \in \boldsymbol{R}^{n \times n^{2}}
$$

is the reachability matrix of (14), where

$$
B_{1}=\bar{B}_{x}^{-1}\left(\bar{E}_{c}^{0}-\bar{C}_{x}^{0} \bar{B}_{x}^{-1} B_{y}\left(I_{m}-W_{y}\right)^{-1} W_{c}\right) ; B_{2}=\bar{B}_{x}^{-1} \bar{E}_{c}^{0}
$$

Then, any given prefixed production vector $x^{*} \succeq \min \left(\left(\bar{B}_{x}^{-1} \bar{C}_{x}^{0}\right)^{n} x_{t}+B_{0} c_{t+n}, 0\right)$ is reachable with $u_{(.)} \equiv 0$ at the $(t+n)$-sample by a vector of demands

$$
\bar{c}_{t}=\left(R_{c} R_{c}^{T}\right)^{-1} R_{c}\left(x^{*}-\left(\bar{B}_{x}^{-1} \bar{C}_{x}^{0}\right)^{n} x_{t}-B_{0} c_{t+n}\right) \succeq 0
$$

Proof. One gets from (14) that for any $t \in Z_{0+}$ :

$$
\begin{gathered}
x_{t+1}=\bar{B}_{x}^{-1} \bar{C}_{x}^{0} x_{t}+\bar{B}_{x}^{-1} \bar{E}_{c}^{0} c_{t}-\bar{B}_{x}^{-1} B_{y}\left(I_{m}-W_{y}\right)^{-1} W_{c} c_{t+1} \\
x_{t+2}=\bar{B}_{x}^{-1} \bar{C}_{x}^{0} x_{t+1}+\bar{B}_{x}^{-1} \bar{E}_{c}^{0} c_{t+1}-\bar{B}_{x}^{-1} B_{y}\left(I_{m}-W_{y}\right)^{-1} W_{c} c_{t+2} \\
=\bar{B}_{x}^{-1} \bar{C}_{x}^{0}\left[\bar{B}_{x}^{-1} \bar{C}_{x}^{0} x_{t}+\bar{B}_{x}^{-1} \bar{E}_{c}^{0} c_{t}-\bar{B}_{x}^{-1} B_{y}\left(I_{m}-W_{y}\right)^{-1} W_{c} c_{t+1}\right]+\bar{B}_{x}^{-1} \bar{E}_{c}^{0} c_{t+1}-\bar{B}_{x}^{-1} B_{y}\left(I_{m}-W_{y}\right)^{-1} W_{c} \mathcal{c}_{t+2} \\
=\left(\bar{B}_{x}^{-1} \bar{C}_{x}^{0}\right)^{2} x_{t}+\bar{B}_{x}^{-1} \bar{C}_{x}^{0} \bar{B}_{x}^{-1} \bar{E}_{c}^{0} c_{t}+\bar{B}_{x}^{-1}\left[\bar{E}_{c}^{0}-\bar{C}_{x}^{0} \bar{B}_{x}^{-1} B_{y}\left(I_{m}-W_{y}\right)^{-1} W_{c}\right] c_{t+1}-\bar{B}_{x}^{-1} B_{y}\left(I_{m}-W_{y}\right)^{-1} W_{c} c_{t+2} \\
x_{t+i}=\left(\bar{B}_{x}^{-1} \bar{C}_{x}^{0}\right)^{i} x_{t}+\sum_{j=0}^{i-1}\left(\bar{B}_{x}^{-1} \bar{C}_{x}^{0}\right)^{i-j-1}\left(\bar{B}_{x}^{-1} \bar{E}_{c}^{0} c_{t+j}-\bar{B}_{x}^{-1} B_{y}\left(I_{m}-W_{y}\right)^{-1} W_{c} c_{t+j+1}\right) \\
x_{t+n}=\left(\bar{B}_{x}^{-1} \bar{C}_{x}^{0}\right)^{n} x_{t}+\sum_{j=0}^{n-1}\left(\bar{B}_{x}^{-1} \bar{C}_{x}^{0}\right)^{n-j-1} \bar{B}_{x}^{-1} \bar{E}_{c}^{0} c_{t+j} \\
-\sum_{j=1}^{n}\left(\bar{B}_{x}^{-1} \bar{C}_{x}^{0}\right)^{n-j} \bar{B}_{x}^{-1} B_{y}\left(I_{m}-W_{y}\right)^{-1} W_{c} c_{t+j} \\
=\left(\bar{B}_{x}^{-1} \bar{C}_{x}^{0}\right)^{n} x_{t}+\sum_{j=1}^{n-1}\left(\bar{B}_{x}^{-1} \bar{C}_{x}^{0}\right)^{n-j-1}\left(\bar{B}_{x}^{-1} \bar{E}_{c}^{0}-\bar{B}_{x}^{-1} \bar{C}_{x}^{0} \bar{B}_{x}^{-1} B_{y}\left(I_{m}-W_{y}\right)^{-1} W_{c}\right) c_{t+j} \\
+\left(\bar{B}_{x}^{-1} \bar{C}_{x}^{0}\right)^{n-1} \bar{B}_{x}^{-1} \bar{E}_{c}^{0} c_{t}-\bar{B}_{x}^{-1} B_{y}\left(I_{m}-W_{y}\right)^{-1} W_{c} c_{t+n}
\end{gathered}
$$

so that 


$$
\begin{aligned}
& x_{t+n}-\left(\bar{B}_{x}^{-1} \bar{C}_{x}^{0}\right)^{n} x_{t}=\sum_{j=1}^{n-1}\left(\bar{B}_{x}^{-1} \bar{C}_{x}^{0}\right)^{n-j-1}\left(\bar{B}_{x}^{-1} \bar{E}_{c}^{0}-\bar{B}_{x}^{-1} \bar{C}_{x}^{0} \bar{B}_{x}^{-1} B_{y}\left(I_{m}-W_{y}\right)^{-1} W_{c}\right) c_{t+j} \\
& +\left(\bar{B}_{x}^{-1} \bar{C}_{x}^{0}\right)^{n-1} \bar{B}_{x}^{-1} \bar{E}_{c}^{0} \mathcal{C}_{t}-\bar{B}_{x}^{-1} B_{y}\left(I_{m}-W_{y}\right)^{-1} W_{c} \mathcal{c}_{t+n} \\
& =\bar{R}_{c} \bar{c}_{t}^{0}
\end{aligned}
$$

$$
\begin{gathered}
\text { where } \bar{c}_{t}^{0}=\left(c_{t+n}^{T}, c_{t+n-1}^{T}, \ldots, c_{t}^{T}\right)^{T}=\left(c_{t+n}^{T}, \bar{c}_{t}^{T}\right)^{T} \in \boldsymbol{R}^{(n+1) n}, \text { and } \\
\bar{R}_{c}=\left[B_{0}, R_{c}\right]=\left[B_{0}, B_{1},\left(\bar{B}_{x}^{-1} \bar{C}_{x}^{0}\right) B_{1}, \cdots,\left(\bar{B}_{x}^{-1} \bar{C}_{x}^{0}\right)^{n-2} B_{1},\left(\bar{B}_{x}^{-1} \bar{C}_{x}^{0}\right)^{n-1} B_{2}\right] \in \boldsymbol{R}^{n \times(n+1) n}
\end{gathered}
$$

with

$$
B_{0}=-\bar{B}_{x}^{-1} B_{y}\left(I_{m}-W_{y}\right)^{-1} W_{c}
$$

so that (27) is equivalent to:

$$
x_{t+n}-\left(\bar{B}_{x}^{-1} \bar{C}_{x}^{0}\right)^{n} x_{t}-B_{0} c_{t+n}=R_{c} \bar{c}_{t}
$$

The algebraic system in the unknown vector $\bar{c}_{t}$, which is obtained by deleting $c_{t+n}$ in the unknown vector $\bar{c}_{t}^{0}$ driving (27), allows to overcome a requirement of $B_{0} \succ 0$ in the extended reachability matrix (28) of difficult generic compatibility with the assumption 4 in view of (22). Thus, $c_{t+n}$ can be prefixed to a generic non-negative value, just being subject to a rank condition specified later on, without fixing its value being a handicap for the reachability of a prefixed targeted production vector on the time interval $[t, t+n]$ being achievable through the choice of $c_{t}$. Note also that $x_{t+n}$ can be fixed to any given prefixed $x^{*}$ at the sample $t+n$ from any initial condition $x_{t}$ by some vector of demands $\bar{c}_{t}$ if and only if the reachability matrix defined by (22) is of full row rank $n$, which is equivalent to the controllability matrix to be full rank. This is because, resulting from the Rouché-Capelli theorem, one has in that case that,

$$
n=\operatorname{rank}\left(R_{c}\right)=\operatorname{rank}\left(R_{c}, x^{*}-\left(\bar{B}_{x}^{-1} \bar{C}_{x}^{0}\right)^{n} x_{t}-B_{0} c_{t+n}\right)
$$

which, according to the rank condition in the assumption 5 of the theorem, ensures that the algebraic system (30) has at least a demand vector solution $\bar{c}_{t}$ for any prefixed production vector $x^{*}$ subject to any initial condition $x_{t}$. Until now, it has not been invoked that the system has to be productive, that is, $\bar{c}_{t} \succ 0$ for any given targeted production vector $x^{*} \succeq \min \left(\left(\bar{B}_{x}^{-1} \bar{C}_{x}^{0}\right)^{n} x_{t}+B_{0} c_{t+n}, 0\right)$, subject to any initial condition $x_{t} \succeq 0$. Note that:

(a) $\quad R_{c} \succ 0$ from the assumptions (1-4) and in view of (22)-(24),

(b) It exists $\left(R_{c} R_{c}^{T}\right)^{-1} \succ 0$, since it is diagonal with positive diagonal entries (then also monomial) since $R_{c}$ has only a positive entry per row and it is of full row rank,

(c) In view of (31) the set of solutions $\bar{c}_{t} \in R^{n^{2}}$ of (30) may be calculated with the Moore-Penrose pseudoinverse of $R_{c}$, i.e., $R_{c}^{+}=\left(R_{c} R_{c}^{T}\right)^{-1} R_{c} \succ 0$ as

$$
\bar{c}_{t}=\bar{c}_{t}(w)=R_{c}^{\dagger}\left(x^{*}-\left(\bar{B}_{x}^{-1} \bar{C}_{x}^{0}\right)^{n} x_{t}-B_{0} c_{t+n}-R_{c} w\right)+w
$$

being parametric on the arbitrary vector $w \in \boldsymbol{R}^{n^{2}}$. If $w \succeq 0$ and $x^{*} \succeq$ $\min \left(\left(\bar{B}_{x}^{-1} \bar{C}_{x}^{0}\right)^{n} x_{t}+B_{0} c_{t+n}+R_{c} w, 0\right)$ then $\bar{c}_{t} \succeq 0$ is obtained from (32). The specific result of the theorem follows for the choice $w=0$ to achieve a production objective $x_{t+n}=x^{*} \succeq \min \left(\left(\bar{B}_{x}^{-1} \bar{C}_{x}^{0}\right)^{n} x_{t}+B_{0} c_{t+n}, 0\right)$ through the extended vector of demands $\bar{c}_{t}=\bar{c}_{t}(0)=R_{c}^{\dagger}\left(x^{*}-\left(\bar{B}_{x}^{-1} \bar{C}_{x}^{0}\right)^{n} x_{t}-B_{0} c_{t+n}\right)$. Since $R_{c} \succ 0$ and $\bar{c}_{t} \succeq 0$, it also turns from (30) that any product obtained by deleting block columns of $R_{c}$ and 
the corresponding sub-vectors of $\bar{c}_{t}$ is also non-negative. Thus, $x_{t+i}-\left(B_{x}^{-1} \bar{C}_{x}^{0}\right)^{i} x_{t}-$ $B_{0} c_{t+i} \succeq 0$ which implies that $x_{t+i} \succeq 0$ for $i=1,2, \ldots, n$ since $x_{t} \succeq 0, \bar{c}_{t} \succ 0$ and $x_{t+n}=x^{*} \succeq 0$.

The reachability conditions of Theorem 1 can be sometimes relaxed by involving less demand components than those integrating the vector of demands $\bar{c}_{t}$ on $[t, t+n-1]$. Define a reduced vector of demands $\bar{c}_{t}=Q \bar{c}_{t}^{r e d}$ with $\bar{c}_{t}^{\text {red }} \in \boldsymbol{R}^{q}, Q \in \boldsymbol{R}_{+}^{n^{2} \times q}$ for some integer $n \leq q \leq n^{2}$. The reduction matrix $Q=Q(q)$ expresses the vector of demands on the integer interval $[t, t+n-1]$ as a combination of components of the reduced vector of demands. Note that:(1) $q \geq n$ since, otherwise, the reachability problem could not be algebraically solved with less than $n$ unknowns to compute the vector of demands in (28) such that $x_{t+n}=x^{*} \succeq 0$; (2) some combinations of demands through time could potentially satisfy targeting a prefixed production at $(t+n)$ without the need of determining each demand component in an isolated way; (3) on occasions, it can happen that a demand at some service can collapse, for instance, as a result of a terrorist attack, bankruptcy, strikes, or a serious accident in certain consumptions sector; (4) the reduction matrix may be nonunique, in general, and it can be sparse. The above considerations lead to the subsequent corollary of Theorem 1, whose proof is direct from that of Theorem 1.

Corollary 1. (size-reduced reachability matrix). Assume that the assumptions 1-4 of Theorem 1 hold and also that the assumption 5 is modified to rank $\left(R_{c}^{r e d}\right)=n$ for some size-reduced reachability matrix $R_{c}^{r e d}$ defined based on (31) via some prefixed full rank $Q \in \boldsymbol{R}_{+}^{n^{2} \times q}$ for some integer $n \leq q \leq n^{2}$ as follows:

$$
R_{c}^{r e d}=\left[B_{1} Q,\left(\bar{B}_{x}^{-1} \bar{C}_{x}^{0}\right) B_{1} Q, \cdots,\left(\bar{B}_{x}^{-1} \bar{C}_{x}^{0}\right)^{n-2} B_{1} Q,\left(\bar{B}_{x}^{-1} \bar{C}_{x}^{0}\right)^{n-1} B_{2} Q\right] \in \boldsymbol{R}^{n \times q}
$$

Then, any given prefixed production vector $x^{*} \succeq\left(\bar{B}_{x}^{-1} \bar{C}_{x}^{0}\right)^{n} x_{t}+Q B_{0} c_{t+n}$ is reachable at the $(t+n)$-sample by a reduced vector of demands:

$$
\bar{c}_{t}^{\text {red }}=R_{c}^{\text {red }+}\left(x^{*}-\left(\bar{B}_{x}^{-1} \bar{C}_{x}^{0}\right)^{n} x_{t}-B_{0} c_{t+n}\right)
$$

from any given initial condition $x_{t} \succeq 0$ at the $t$-sample.

Note that by taking into account the definitions of the auxiliary matrices $E_{c}^{0}, \bar{E}_{c}^{0}$, and $\bar{B}_{x}$ in (15), then the matrices $B_{1}, B_{2}$, and $B_{0},(23)$ and (29) become expanded as follows when related to the primary parameterization of the uncontrolled model, i.e., for $u \equiv 0$ in (4):

$$
\begin{gathered}
B_{1}=\left[B_{x}+B_{y}\left(I_{m}-W_{y}\right)^{-1} W_{x}\right]^{-1}\left[\left(D_{y}^{0}-\bar{C}_{x}^{0} \bar{B}_{x}^{-1} B_{y}\right)\left(I_{m}-W_{y}\right)^{-1} W_{c}-I_{n}\right] \\
B_{2}=\left[B_{x}+B_{y}\left(I_{m}-W_{y}\right)^{-1} W_{x}\right]^{-1}\left[D_{y}^{0}\left(I_{m}-W_{y}\right)^{-1} W_{c}-I_{n}\right] \\
B_{0}=-\left[B_{x}+B_{y}\left(I_{m}-W_{y}\right)^{-1} W_{x}\right]^{-1} B_{y}\left(I_{m}-W_{y}\right)^{-1} W_{c}
\end{gathered}
$$

Remark 3. The non-negativity conditions like, for instance, $\left(x^{*}-\left(\bar{B}_{x}^{-1} \bar{C}_{x}^{0}\right)^{n} x_{t}-B_{0} c_{t+n}\right) \succeq 0$ and $\bar{c}_{t} \succeq 0$ in Theorem 1 and Corollary 1 are used for addressing mathematical formalism only. In practice, the non-negativity conditions have to be replaced by positive, respectively, by strictly positive ones, in order for the model to be productive in at least one sector, respectively, in all sectors.

Remark 4. Note from the complete and reduced reachability matrices (22) and (33) and the CayleyHamilton theorem from Linear Algebra that if the degree of the minimal polynomial of $\bar{B}_{x}^{-1} \bar{C}_{x}^{0}$ is $1 \leq \mu<n$ and, since the powers of a matrix exceeding the degree of its minimal polynomial 
satisfy the constraint of its characteristic equation (Cayley-Hamilton theorem), any matrices of the form $\left(\bar{B}_{x}^{-1} \bar{C}_{x}^{0}\right)^{j}$ for $j \geq \mu$ can be expressed as a linear combination of the matrices $\left(\bar{B}_{x}^{-1} \bar{C}_{x}^{0}\right)^{k}$ for $k=0,1, \ldots, \mu-1$. Therefore, Corollary 1 can be reformulated mutatis-mutandis for a lower-dimensioned reachability matrix and reduced vector of demands according to $\mu \leq q \leq \mu^{2}$.

Now, consider the controlled model (1)-(3), subject to the special dyadic form $K_{x}=b \hat{k}_{x}^{T}$ of the matrix $K_{x}$, where $b \in \boldsymbol{R}^{n}, \hat{k}_{x} \in \boldsymbol{R}^{n}$ with $K_{y}=0$ leading to the following particular from of the feedback control of (4):

$$
u_{t}=-b \hat{k}_{x}^{T} x_{t}-K_{c} c_{t}
$$

If the above control law is used in (19) and (12) and (15)-(17), then the system evolves according to

$$
\begin{gathered}
x_{t+1}=\bar{B}_{x}^{-1}\left(\bar{C}_{x}^{0}+b \hat{k}_{x}^{T}\right) x_{t}+\bar{B}_{x}^{-1}\left[\left(K_{c}+\bar{E}_{c}^{0}\right) c_{t}-B_{y}\left(I_{m}-W_{y}\right)^{-1} W_{c} c_{t+1}\right] \\
=\bar{B}_{x}^{-1} \bar{C}_{x}^{0} x_{t}+\bar{B}_{x}^{-1} b \hat{k}_{x}^{T} x_{t}+g_{t}
\end{gathered}
$$

where

$$
g_{t}=\bar{B}_{x}^{-1}\left[\left(K_{c}+\bar{E}_{c}^{0}\right) c_{t}-B_{y}\left(I_{m}-W_{y}\right)^{-1} W_{c} c_{t+1}\right]
$$

Then, Equation (27), or Equation (30), becomes modified as follows by using (36) and (37):

$$
\begin{aligned}
\hat{x}_{t+n} & :=x_{t+n}-\left(\bar{B}_{x}^{-1} \bar{C}_{x}^{0}\right)^{n} x_{t}-\sum_{j=0}^{n-1}\left(\bar{B}_{x}^{-1} \bar{C}_{x}^{0}\right)^{n-j-1} g_{t+j} \\
& =\sum_{j=0}^{n-1}\left(\bar{B}_{x}^{-1} \bar{C}_{x}^{0}\right)^{n-j-1} \bar{B}_{x}^{-1} b \hat{k}_{x}^{T} x_{t+j}=R_{c b} \bar{\vartheta}_{t}
\end{aligned}
$$

where $u_{t+j}=-b \hat{k}_{x}^{T} x_{t+j} ; j=0,1, \ldots, n, \bar{\vartheta}_{t}=\left(\vartheta_{t+n-1}, \ldots, \vartheta_{t}\right)^{T}=\left(\hat{k}_{x}^{T} x_{t+n-1}, \ldots, \hat{k}_{x}^{T} x_{t}\right)^{T}$ and

$$
R_{c b}=\left[\bar{B}_{x}^{-1} b,\left(\bar{B}_{x}^{-1} \bar{C}_{x}^{0}\right) \bar{B}_{x}^{-1} b, \cdots,\left(\bar{B}_{x}^{-1} \bar{C}_{x}^{0}\right)^{n-2} \bar{B}_{x}^{-1} b,\left(\bar{B}_{x}^{-1} \bar{C}_{x}^{0}\right)^{n-1} \bar{B}_{x}^{-1} b\right] \in \boldsymbol{R}^{n \times n}
$$

Thus, the following result holds:

Theorem 2. (fixing the model dynamics via feedback). Assume that the assumptions 1-4 of Theorem 1 hold and that: (6) The reachability matrix $R_{c b}$ has only a nonzero entry per row and $\operatorname{rank}\left(R_{c b}\right)=n$, equivalently, the pair $\left(\bar{B}_{x}^{-1} \bar{C}_{x}^{0}, \bar{B}_{x}^{-1} b\right)$ is reachable.

Assume also that the control law (36) is applied with $\hat{k}_{x} \in \boldsymbol{R}^{n}$ fixed as follows:

$$
\hat{k}_{x}=[0,0, \cdots, 1] R_{c b}^{-1} \alpha_{c}\left(\bar{B}_{x}^{-1} \bar{C}_{x}^{0}\right)
$$

where

$$
\alpha_{c}(z)=\operatorname{det}\left(z I_{n}-\bar{B}_{x}^{-1} \bar{C}_{x}^{0}-\bar{B}_{x}^{-1} b \hat{k}_{x}^{T}\right)=z^{n}-\sum_{i=1}^{n} \alpha_{i} z^{n-i}
$$

for any given set of real prefixed coefficients $\alpha_{i} \in \boldsymbol{R} ; i \in \bar{n}$. Then, the following properties hold:

(i) The closed-loop characteristic polynomial of the system (1)-(3), under the control law (36) subject to (40) and under the assumptions 1-4 of Theorem 1 together with the assumptions 6 is given by (42).

(ii) The closed-loop system has a pole of order $n$ at $z=\alpha$ if $\hat{k}_{x n}=1+a_{n}$ and $\hat{k}_{x i}=a_{i}+$ $\left(\begin{array}{c}n \\ i\end{array}\right) \alpha^{i} ; i=1,2, \ldots, n-1$. If $|\alpha|<1$ then the closed-loop system is stable and $|\alpha|$ is the spectral radius of its closed-loop matrix of dynamics $\bar{B}_{x}^{-1} \bar{C}_{x}^{0}+\bar{B}_{x}^{-1} b \hat{k}_{x}^{T}$. 
Proof. It turns out that fixing (41) into the control law (36) gives a prefixed closed-loop characteristic polynomial (42). This conclusion follows since:

(a) The square matrix defining the closed-loop dynamics satisfies its own characteristic polynomial, from Cayley-Hamilton theorem after replacing $z \rightarrow \bar{B}_{x}^{-1} \bar{C}_{x}^{0}-\bar{B}_{x}^{-1} b \hat{k}_{x}^{T}$ in (42), leading to

$$
\alpha_{c}\left(\bar{B}_{x}^{-1}\left(\bar{C}_{x}^{0}-b \hat{k}_{x}^{T}\right)\right)=\left(\bar{B}_{x}^{-1}\left(\bar{C}_{x}^{0}-b \hat{k}_{x}^{T}\right)\right)^{n}-\sum_{i=1}^{n} \alpha_{i}\left(\bar{B}_{x}^{-1}\left(\bar{C}_{x}^{0}-\bar{B}_{x}^{-1} b \hat{k}_{x}^{T}\right)\right)^{n-i}
$$

(b) Since the system is reachable, the pair $\left(\bar{B}_{x}^{-1} \bar{C}_{x}^{0}, \bar{B}_{x}^{-1} b\right)$ can be transformed to the controller form

$$
\Phi=T^{-1} \bar{B}_{x}^{-1} \bar{C}_{x}^{0} T=\left[\begin{array}{cccc}
a_{1} & a_{2} & \cdots & a_{n} \\
1 & 0 & \cdots & 0 \\
0 & 1 & 0 \cdots & 0 \\
0 & \cdots & 0 & 10
\end{array}\right] ; \Gamma=T^{-1} b=\left[\begin{array}{c}
1 \\
0 \\
\vdots \\
0
\end{array}\right]
$$

with characteristic polynomial of both $\Phi$ and $\bar{B}_{x}^{-1} \bar{C}_{x}^{0}$ being

$$
a(z)=\operatorname{det}\left(z I_{n}-\bar{B}_{x}^{-1} \bar{C}_{x}^{0}\right)=z^{n}-\sum_{i=1}^{n} a_{i} z^{n-i}
$$

which is the open-loop characteristic equation of the system, that is, in the absence of control with $\hat{k}_{x}=0$,

(c) After feedback control via (36), subject to the control gain (41), resulting in being identical to

$$
\hat{k}_{x}=\left(\hat{k}_{x 1}, \hat{k}_{x 2}, \cdots, \hat{k}_{x n}\right)^{T}=\left(a_{1}-\alpha_{1}, a_{2}-\alpha_{2}, \cdots, a_{n}-\alpha_{n}\right)^{T} \in \boldsymbol{R}^{n}
$$

from Ackermann's formula [35]. This fact implies that the closed-loop system characteristic equation becomes modified from $a(z)=0$ to (see (45)):

$$
a_{c}(z)=\operatorname{det}\left(z I_{n}-\bar{B}_{x}^{-1} \bar{C}_{x}^{0}+\bar{B}_{x}^{-1} b \hat{k}_{x}^{T}\right)=z^{n}-\sum_{i=1}^{n} \alpha_{i} z^{n-i}=z^{n}-\sum_{i=1}^{n}\left(a_{i}-\hat{k}_{x i}\right) z^{n-i}=0
$$

Property (i) has been proved. To prove Property (ii) note from (47) that all its zeros are re-allocated at $z=\alpha \in(-1,1)$ with the control law (36), from their initial positions at the zeros of $a(z)$, if $a_{c}(z)=(z-\alpha)^{n}$ which equalizes $a_{c}(z)=z^{n}-\sum_{i=1}^{n} \alpha_{i} z^{n-i}$ by equating the corresponding coefficients of the powers of $z$ yields $\alpha_{0}=1, \alpha_{n}=(-1)^{n} \alpha^{n}$ and $\alpha_{i}=-\left(\begin{array}{c}n \\ i\end{array}\right) \alpha^{i} ; i=1,2, \ldots, n-1$. Thus, one gets $\hat{k}_{x n}=a_{n}-\alpha_{n}$ and $\hat{k}_{x i}=a_{i}+\left(\begin{array}{c}n \\ i\end{array}\right) \alpha^{i} ;$ $i=1,2, \ldots, n-1$ by using (46). All the eigenvalues of $\bar{B}_{x}^{-1}\left(\bar{C}_{x}^{0}-b \hat{k}_{x}^{T}\right)$ are re-allocated at $z=\alpha$, and are stable if $|\alpha|<1$, by the control law from their positions at the zeros of (45) in the absence of control. Property (ii) has been proved.

Remark 5. Note that the initial production consumption and the values of the demand sequence are irrelevant for the fulfilment of Theorem 2 (rather than for the obtained solution), since (39) is solvable irrespective of its left-hand-side value if the reachability matrix is full rank.

Remark 6. Note that Theorem 2 addresses a method for closed-loop pole placement under the control law (36) provided that the uncontrolled system is reachable. The closed-loop characteristic equation is dealt with by using the companion canonical controllability form of the system which is not, in general, associated with the initial space time realization which, by virtue of the nature of this problem has to be (internally) positive, i.e., all its state variables have to be non-negative for all time under non-negative inputs. It is well-known in the field of positive systems that the positivity 
is not, in general, preserved under state-space transformations [19,29-31]. Therefore, it is needed for well-posedness reasons to ensure that the closed-loop pole-placement assignment is such that the positivity of the initial closed-loop state-space realization is preserved.

The subsequent result relies on the comments in Remark 6 for positivity of the closedloop system (13) under the control law (36).

Theorem 3. (non-negativity of the solution). Assume that Assumption 1 holds, $\left(I_{m}-W_{y}\right)^{-1} \succ 0$ exists, i.e., if either $W_{y} \succeq 0$ is monomial or $W_{y}$ is a non-singular $M$-matrix (i.e., it is an M-matrix with positive principal minors) and, furthermore, $K_{y}=0, K_{x}=b \hat{k}_{x}^{T}$ and $K_{c}$ are such that Theorem 2 holds, and, in addition,

$$
\begin{gathered}
K_{c} \succeq B_{y}\left(I_{m}-W_{y}\right)^{-1} W_{c} \sigma_{t}+I_{n}-D_{y}\left(I_{m}-W_{y}\right)^{-1} W_{c} \\
\left(B_{x}+B_{y}\left(I_{m}-W_{y}\right)^{-1}\right) \min \left(\sigma_{t},\left(I_{n}+B_{x}-A_{x}+b \hat{k}_{x}^{T}+\left(B_{y}+P-A_{y}\right)\left(I_{m}-W_{y}\right)^{-1} W_{x}\right)\right) \succeq 0 \\
\text { where } \sigma_{t}=c_{t+1} / c_{t} \text { with } c_{t} \succ 0 \text { for all } t \in Z_{0+} . \\
\text { Then, (13) and (18) have non-negative solutions. }
\end{gathered}
$$

Proof. Note by direct calculations that (13), with the parameterization (15)-(17) subject to (7)-(9), and (18) have non-negative solutions under the conditions (48) and (49).

Concerning Theorem 3, note that, if the open-loop, that is uncontrolled, system is internally positive, then (48) is guaranteed if $K_{c} \succ \succ 0$ with sufficiently large entries. Additionally, if the open-loop system satisfies strictly (49) which implies that (49) holds for $\hat{k}_{x}=0$ then, there is some range of values $\left[-\hat{k}_{x m}, \hat{k}_{x M}\right]$ where (49) still holds for $\hat{k}_{x} \in\left[-\hat{k}_{x m}, \hat{k}_{x M}\right]$.

\section{Extensions of the Results to the General Singular Regular Leontief Model}

This section relies on the extension of the study of reachability without the nonsingularity constraint on re-usable goods. It is of interest to extend the above study to the case when (5) satisfies $\operatorname{det}(\gamma E-A) \neq 0$ for some complex number $\gamma$ so that the whole model is referred to as being "regular", despite $E$ being singular in the model (5), subject to (6)-(9), whose open-loop version is (10), subject to (11) and (12). In such a case, the system is said to be singular regular. See, for instance $[2,8,19,25-29]$. The singularity problem was dealt with for the standard Leontief models of [8]. In this way, this section is devoted to the more general controlled model (5)-(9) of Section 2 and its open-loop counterpart (10)-(12). For further generalization purposes, it is assumed that, in general, $I_{m}-W_{y}$ might be singular.

By convenience, we can describe the closed-loop system (5) by re-entering the feedback law as a forcing contribution, using (12) as follows:

$$
E z_{t+1}=\left(A^{0}+K\right) z_{t}+B c_{t}
$$

where

$$
K=\left[\begin{array}{ccc}
K_{x_{n \times n}} & K_{y_{n \times m}} & 0_{n \times o} \\
0_{m \times n} & 0_{m \times m} & 0_{m \times o} \\
0_{o \times n} & 0_{o \times m} & 0_{o \times o}
\end{array}\right] ; B=\left[\begin{array}{c}
E_{c} \\
W_{c} \\
0_{o \times n}
\end{array}\right]
$$

Therefore, the z- transform under zero initial conditions of the whole model (5), a compact version of (1)-(4), whose state vector includes, in the most general case, nonrenewable, reusable, and recycled products, becomes:

$$
Z(z)=(z E-A)^{-1} B C(z)=\left(z E-A^{0}-K\right)^{-1} B_{c} C(z)
$$


where $B_{c}=\left[K_{c}^{T}-I_{n}, W_{c}^{T}, 0_{n \times o}\right]^{T}$ and $C(z)$ is the $z$-transform of the sequence $c_{t}$. The system (5) is regular if and only if there exists a complex number $\gamma$ such that $\operatorname{det}(\gamma E-A)=$ $\operatorname{det}\left(\gamma E-A^{0}-K\right) \neq 0$. It is well-known that the system is solvable if it is regular. Since such a property depends on the pairs $\left(E, A^{0}\right)$ for the open-loop system (10) and on the pair $(E, A)$ for the closed-loop system (5), it is said that:

(a) the open-loop (i.e., uncontrolled) system is solvable if the pair $\left(E, A^{0}\right)$ is regular,

(b) the closed-loop (i.e., controlled) system is solvable if the pair $(E, A)$ is regular.

The following result holds $[2,28,29]$ :

Theorem 4. (regularity conditions of the singular system). The following properties hold:

(i) Refs. [2,28,29]. Define the following matrix:

$$
G(k)=\left\langle\begin{array}{c}
E \\
A
\end{array}\right\rangle:=\left[\begin{array}{cccc}
E & & & \\
A & E & & \\
& A & \ddots & \\
& & \ddots & E \\
& & & A
\end{array}\right] \in R^{(k+1)(n+m+o) \times(n+m+o) k}
$$

Then, the pair $(E, A)$ is regular if and only if $\operatorname{rank} G(k)=n k, \forall k \in \mathbf{Z}_{+}$.

(ii) Refs. [2,28,29]. Define the following matrix

$$
F(k)=\left\langle\begin{array}{ll}
E & A
\end{array}\right\rangle:=\left[\begin{array}{cccc}
E & A & & \\
& E & A & \\
& & \ddots & \\
& & \ddots & A \\
& & & E A
\end{array}\right] \in R^{(n+m+o) k \times(n+m+o)(k+1)}
$$

Then, the pair $(E, A)$ is regular if and only if $\operatorname{rank} F(k)=(n+m+o) k$.

(iii) Ref. [28]. The pair $(E, A)$ is regular if and only if $\max \left\{\operatorname{rank}\left(s_{i} E-A\right) ; i \in \bar{\ell}\right\}=n+$ $m+o$ for any arbitrarily selected set of distinct complex numbers $s_{i} \in C ; i \in \bar{\ell}$ where $\ell=\operatorname{deg} \operatorname{det}(s E-A)$.

(iv) Ref. [28]. Assume that $\operatorname{rank}(E)=n_{0}$ and that $P, Q \in \boldsymbol{R}^{(n+m+o) \times(n+m+o)}$ are nonsingular matrices which satisfy: $Q E P=\operatorname{BlockDiag}(\hat{E}, 0)$ and $Q A P=\hat{A}=$ Block Matrix $\left(\hat{A}_{i j} ; i, j=1,2\right)$. Then, $(E, A)$ is regular if and only if

$$
\operatorname{det}\left(\hat{A}_{22}+\hat{A}_{21}\left(z \hat{E}-\hat{A}_{11}\right)^{-1} A_{12}\right) \neq 0
$$

where $Z\left(\hat{E}, \hat{A}_{11}\right):=\left\{z \in C: \operatorname{det}\left(z \hat{E}-\hat{A}_{11}\right)=0\right\}$.

Note that non-singular $(n+m+o)$-matrices $P$ and $Q$ of Theorem 4 (iv) always exist, leading to an equivalence transformation to a diagonal form $E_{d} \approx Q E P=\operatorname{BlockDiag}(\hat{E}, 0)$ with $\hat{E}$ being full rank. A particular equivalence transformation is that leading to $\operatorname{BlockDiag}(\hat{E}, 0)$ being the canonical Smith form $[2,8]$, of $E$ such that $\hat{E}$ is diagonal with its diagonal entries being the invariant factors of $E$ (such that each one of them divides that of the next adjacent diagonal entry). It is directly observable that by redefining $P \rightarrow P^{\prime}=M^{-1} P$, with $M^{-1}$ being a diagonal normalization matrix of the canonical Smith form, such a Smith form becomes equivalent to a matrix whose main diagonal block is an identity matrix of order equal to the rank of $E$ and the other diagonal entries being zero.

Another equivalent matrix can be obtained in the so-called canonical KroneckerWeirstrass form, where $E$ is transformed to a block-diagonal matrix with a non-singular matrix block plus a nilpotent one $[2,28]$. However, a similarity transform, that is with 
$T=P=Q^{-1}$ of $E$ to $\operatorname{BlockDiag}(\hat{E}, 0)=T^{-1} E T$, does not always exist with just diagonal entries and zero off-diagonal entries, with $\hat{E}$ being full rank, since $E$ can be degenerate with eigenvalues of multiplicities larger than one. In that case, the similar matrix would be, in general, triangular, rather than diagonal. On the other hand, a similarity transformation exists [36,37], via $T=P=Q^{-1}$ of $E$ to $\operatorname{BlockDiag}(\hat{E}, \hat{N})=T^{-1} E T$, where $\hat{E}$ and $\hat{N}$ are Jordan matrix blocks (in general, non being purely diagonal) with $\hat{N}$ being a nilpotent matrix.

However, the main problem which arises with any of the above-mentioned, either the equivalence or the similarity transformation, is that the positivity properties of the solution and parameterizing parameters become, in general, lost. Therefore, those described transformations can be useful to investigate the stability properties or simple forms of the solutions under coordinates changes but, in general, they are not directly useful to investigate the positivity properties.

The following result is obvious from Theorem 4(i) and it can be extended easily from Theorem 4(ii) and Theorem 4(iii). It relies on the regularity of $(E, A)$, provided that $\left(E, A^{0}\right)$ is regular and vice-versa.

Corollary 2. Assume that $G_{0}(k)=\left\langle\begin{array}{c}E \\ A^{0}\end{array}\right\rangle ; \forall k \in Z_{+}$and define $\widetilde{G}(k)=$ $\left\langle\begin{array}{c}0_{(n+m+o) \times(n+m+o)} \\ A-A^{0}\end{array}\right\rangle=G(k)-G_{0}(k) ; \forall k \in Z_{+}$. Thus, the following properties hold:

(i) Assume that $\|\widetilde{G}(k)\|_{2} \leq \varepsilon_{\widetilde{G}}(k)\left\|G_{0}(k)\right\|_{2} ; \forall k \in Z_{+}$such that $\varepsilon_{\widetilde{G}}(k) \in$ $\left[0, \min \left(1, \frac{\lambda_{\min }\left(G_{0}^{T}(k) G_{0}(k)\right)}{\left\|G_{0}(k)\right\|_{2}\left(\left\|G_{0}(k)\right\|_{2}+2\right)}\right)\right) ; \forall k \in Z_{+}$, where $\lambda_{\text {min }}($.$) denotes the minimum eigen-$ value of the real symmetric matrix $($.$) . Then, the pair (E, A)$ is regular if the pair $\left(E_{0}, A\right)$, is regular.

(ii) Assume that $\left\|\widetilde{G}_{0}(k)\right\|_{2} \leq \varepsilon_{\widetilde{G}_{0}}(k)\|G(k)\|_{2} ; \forall k \in Z_{+}$such that $\varepsilon_{\widetilde{G}_{0}}(k) \in$ $\left[0, \min \left(1, \frac{\lambda_{\min }\left(G^{T}(k) G^{T}(k)\right)}{\|G(k)\|_{2}\left(\|G(k)\|_{2}+2\right)}\right)\right) ; \forall k \in Z_{+}$. Then, the pair $\left(E, A^{0}\right)$ is regular if the pair $(E, A)$, is regular.

Proof. Note that for any given $k \in \boldsymbol{Z}_{+}$the $(n+m+o) k$ square symmetric matrix $G^{T}(k) G(k)$ is positive definite (then non-singular) if and only if $\operatorname{rankF}(k)=(n+m+o) k$, i.e., if and only if $(E, A)$ is regular.

Note that, for any $k \in \boldsymbol{Z}_{+}$, the maximum eigenvalue of $\left(G^{T}(k) G(k)\right), \lambda_{\max }\left(G^{T}(k) G(k)\right)$, is its $\ell_{2}$-norm $\left\|G^{T}(k) G(k)\right\|_{2}$ and that $\|G(k)\|_{2}=\sqrt{\lambda_{\max }\left(G^{T}(k) G(k)\right)}$, and $\lambda_{\text {min }}\left(G^{T}(k) G(k)\right)>0 \Leftrightarrow G^{T}(k) G(k)$ isnon $-\sin$ gular $\Leftrightarrow \operatorname{rank} G(k)=(n+m+o) k$.

Then, direct calculation yields $G^{T}(k) G(k)>0$ for any $k \in Z_{+}$if:

$$
\begin{aligned}
& \lambda_{\min }\left(G^{T}(k) G(k)\right)=\lambda_{\min }\left[\left(G_{0}^{T}(k)+\widetilde{G}^{T}(k)\right)\left(G_{0}(k)+\widetilde{G}(k)\right)\right] \\
& \geq \lambda_{\min }\left(G_{0}^{T}(k) G_{0}(k)\right)-\lambda_{\max }\left(\widetilde{G}^{T}(k) \widetilde{G}(k)+\left(\widetilde{G}^{T}(k) G_{0}(k)+G_{0}^{T}(k) \widetilde{G}(k)\right)\right) \\
& \geq \lambda_{\min }\left(G_{0}^{T}(k) G_{0}(k)\right)-\varepsilon_{\widetilde{G}}(k)\left\|G_{0}(k)\right\|_{2}\left(\varepsilon_{\widetilde{G}}(k)\left\|G_{0}(k)\right\|_{2}+2\right) \\
& \geq \lambda_{\min }\left(G_{0}^{T}(k) G_{0}(k)\right)-\varepsilon_{\widetilde{G}}(k)\left\|G_{0}(k)\right\|_{2}\left(\left\|G_{0}(k)\right\|_{2}+2\right)>0
\end{aligned}
$$

which holds if $\varepsilon_{\widetilde{G}}(k) \in\left[0, \min \left(1, \frac{\lambda_{\min }\left(G_{0}^{T}(k) G_{0}(k)\right)}{\left\|G_{0}(k)\right\|_{2}\left(\left\|G_{0}(k)\right\|_{2}+2\right)}\right)\right)$. Property (i) is proved. Property (ii) is its dual by noting that $G_{0}(k)=G(k)+(-\widetilde{G}(k))$.

Note that the results of Theorem 4 and Corollary 2 only guarantee solvability but not positivity of the solution, which is a requisition for well-posedness. We get the following result on the regularity of $(E, A)$, where $A=A^{0}+K$, provided that $\left(E, A^{0}\right)$ is regular. 
Theorem 5. Assume that $\operatorname{rank}(E)=n_{1}$ and that $P_{0}, Q_{0} \in \boldsymbol{R}^{(n+m+o) \times(n+m+o)}$ are nonsingular matrices which satisfy $Q_{0} E P_{0}=\operatorname{BlockDiag}(\hat{E}, 0)$ and $Q_{0} A^{0} P_{0}=\hat{A}_{0}=$ Block Matrix $\left(\hat{A}_{0 i j} ; i, j=1,2\right)$. Also assume that $\left(E, A^{0}\right)$ is regular.

Then, the following properties hold:

(i) $(E, A)$ is regular if

$$
\left\|\left[\begin{array}{cc}
z \hat{E}-\hat{A}_{011} & -\hat{A}_{012} \\
\hat{A}_{021} & -\hat{A}_{022}
\end{array}\right]^{-1}\left[\begin{array}{cc}
\widetilde{\hat{A}}_{011} & \widetilde{\hat{A}}_{012} \\
\widetilde{\hat{A}}_{021} & \widetilde{\hat{A}}_{022}
\end{array}\right]\right\|<1 ; \forall z \notin Z\left(\hat{E}, \hat{A}_{011}\right)
$$

where $\widetilde{A}=A-A^{0}=K$ and

$$
\widetilde{\hat{A}}=\hat{A}-\hat{A}_{0}=Q_{0}\left(A-A^{0}\right) P_{0}=Q_{0} K P_{0}=Q_{0} \widetilde{A} P_{0}=\hat{\widetilde{A}}=\left[\begin{array}{ll}
\widetilde{\hat{A}}_{11} & \widetilde{\hat{A}}_{12} \\
\widehat{\hat{A}}_{21} & \widetilde{\hat{A}}_{22}
\end{array}\right]
$$

(ii) Assume that $\left(E, A^{0}\right)$ is regular, that $(E, A)$ is guaranteed to be regular according to the conditions of Property $(i)$ and that $\operatorname{rank}(E)=n_{E}<n$. Then, there are some non-singular $P, Q \in \boldsymbol{R}^{n \times n}$ such that $Q E P=\operatorname{BlockDiag}(\hat{E}, 0)$, with $\operatorname{rank}(\hat{E})=n$, and $Q A P=\hat{A}=$ Block Matrix $\left(\hat{A}_{i j} ; i, j=1,2\right)$ such that (53) holds.

Proof. Note that, for $z \notin Z\left(\hat{E}, \hat{A}_{011}\right)$, one has:

$$
\begin{aligned}
& Q_{0}(z E-A) P_{0}=Q_{0}\left(z E-A^{0}-\widetilde{A}\right) P_{0} \\
& =Q_{0}\left(z E-A^{0}\right) P_{0}-Q_{0} \widetilde{A} P_{0} \\
& =\left[\begin{array}{cc}
z \hat{E}-\hat{A}_{011} & -\hat{A}_{012} \\
\hat{A}_{021} & -\hat{A}_{022}
\end{array}\right]+\left[\begin{array}{cc}
\widetilde{\hat{A}}_{11} & \widetilde{\hat{A}}_{12} \\
\widetilde{\hat{A}}_{21} & \widetilde{\hat{A}}_{22}
\end{array}\right] \\
& =\left[\begin{array}{cc}
z \hat{E}-\hat{A}_{011} & -\hat{A}_{012} \\
-\hat{A}_{021} & -\hat{A}_{022}
\end{array}\right]\left(I_{n}-\left[\begin{array}{cc}
z \hat{E}-\hat{A}_{011} & -\hat{A}_{012} \\
-\hat{A}_{021} & -\hat{A}_{022}
\end{array}\right]^{-1}\left[\begin{array}{cc}
\widetilde{\hat{A}}_{11} & \widetilde{\hat{A}}_{12} \\
\widetilde{\hat{A}}_{21} & \widetilde{\hat{A}}_{22}
\end{array}\right]\right)
\end{aligned}
$$

$\forall z \notin Z\left(\hat{E}, \hat{A}_{011}\right)$, since $\left(z \hat{E}-\hat{A}_{011}\right)^{-1}$ exists and since $\left(E, A^{0}\right)$ is regular, what implies from Theorem 4 (iv) that $\left[\begin{array}{cc}z \hat{E}-\hat{A}_{011} & -\hat{A}_{012} \\ -\hat{A}_{021} & -\hat{A}_{022}\end{array}\right]^{-1}$ exists for $z \notin Z\left(\hat{E}, \hat{A}_{011}\right)$, equivalently,

$$
\operatorname{det}\left(\hat{A}_{022}+\hat{A}_{021}\left(z \hat{E}-\hat{A}_{011}\right)^{-1} A_{012}\right) \neq 0 ; z \notin Z\left(\hat{E}, \hat{A}_{011}\right)
$$

One gets from (53) that $(E, A)$ is regular, i.e., $\operatorname{det}(z E-A) \neq 0$ for $z \notin Z\left(\hat{E}, \hat{A}_{011}\right)$, if and only if (53) holds which holds if (57) holds, i.e., if $\left(E, A^{0}\right)$ is regular, provided that (56) also holds.

This also implies that $(z E-A)$ is non-singular for $z \notin Z(E, A)$ so that $(E, A)$ is regular. Property (i) has been proved.

Property (ii) follows since $(E, A)$ is regular if $\left(E, A^{0}\right)$ is regular and, furthermore, (54) holds [Property (i)]. From Theorem 4 (iv), $(E, A)$ is regular if and only if (53) holds for some non-singular $(n+m+o)$-matrices $Q$ and $P$, such that $Q E P=\operatorname{BlockDiag}(\hat{E}, 0)$, with $\operatorname{rank}(\hat{E})=n+m+o$, and $Q A P=\hat{A}=\operatorname{Block} \operatorname{Matrix}\left(\hat{A}_{i j} ; i, j=1,2\right)$.

It is directly by duality that we get the parallel results to Theorem 5 , guaranteeing that $\left(E, A_{0}\right)$ is regular if $(E, A)$ is regular. That is, $\left(E, A^{0}\right)$ is regular if (53) holds (i.e., $(E, A)$ is regular) and (54) holds with $\widetilde{A}_{0}=A^{0}-A=-K$ and the replacements $A^{0} \rightarrow A$ and $\widetilde{\hat{A}}_{0} \rightarrow \widetilde{\hat{A}}$.

We have the following basic result on the standard Kronecker-Weierstrass decomposition of descriptor systems. See, for instance $[2,28]$. It is commonly said that a dynamic system (50) is regular if its corresponding pair $(E, A)$ is regular. 
Lemma 1. (decoupling transformation of a regular system). If $(E, A)$ is regular then there exist non-singular $(n+m+o)$ - matrices $P$ and $Q$, such that the system (50) can be transformed to two decoupled subsystems of dimensions $n_{1}$ and $n_{2}$ with $n+m+o=n_{1}+n_{2}$ of dynamics:

$$
z_{1, t+1}=A_{1} z_{1 t}+B_{1} c_{t} ; N z_{2, t+1}=z_{2 t}+B_{2} c_{t}
$$

where $N \in R^{n_{2} \times n_{2}}$ is a nilpotent matrix of index $\mu$, the same as that of $A=A^{0}+K$, and

$$
\begin{gathered}
\bar{E}=\operatorname{BlockDiag}\left(I_{n_{1}}, N\right)=P E Q, \bar{A}=\operatorname{BlockDiag}\left(A, I_{n_{2}}\right)=P\left(A^{o}+K\right) Q \\
\bar{B}=\left[\begin{array}{c}
\bar{B}_{1} \\
\bar{B}_{2}
\end{array}\right]=P B_{c}
\end{gathered}
$$

with $B_{c}=\left[K_{c}^{T}-I_{n}, W_{c}^{T}, 0_{n \times 0}\right]^{T}$ and the state of the state-space description (50) is $z_{t}=$ $Q\left(z_{1 t}^{T}, z_{2 t}^{T}\right)^{T} ; \forall t \in \mathbf{Z}_{0+}$.

It turns out via recursive calculations that, from boundary mixed initial/final conditions, $x_{10}$, one has from (58) that $z_{2 L}$ for $L \geq n$ :

$$
z_{1 t}=A_{1}^{t} z_{10}+\sum_{i=0}^{t-1} A_{1}^{t-i-1} B_{1} c_{i}, z_{2 t}=N^{L-t} z_{2 L}-\sum_{i=0}^{L-t-1} N^{i} B_{2} c_{t+i} ; \forall t \in \mathbf{Z}_{0+}
$$

and, if $0 \leq t \leq L-\mu$ where $\mu$ is the nilpotency index of $N$ (that is, $N^{\mu-1} \neq 0$ and $N^{\mu}=0$ ) then

$$
z_{2 t}=-\sum_{i=0}^{L-t-1} N^{i} B_{2} c_{t+i}
$$

If $(E, A)$ is regular with $A=A^{0}+K$ then $(z E-A)^{-1}=\sum_{i=-\mu}^{\infty} \Phi_{i} z^{-(i+1)}$, where $\Phi_{i}$ are the fundamental matrices satisfying the relation [19]

$$
E \Phi_{t}-A \Phi_{t-1}=\Phi_{t} E-\Phi_{t-1}\left(A^{0}+K\right)=\left\{\begin{array}{l}
I_{n} \text { if } t=0 \\
0 \text { if } t \neq 0
\end{array} ; \forall t \in Z_{0+}\right.
$$

with $E \Phi_{-\mu}=0$ and $\Phi_{t}=0$ for $t<-\mu$, where $\mu \leq \operatorname{rankE}-\operatorname{deg}(\operatorname{det}(z E-A))+1$ is the nilpotency index of $E$, i.e., $E^{\mu}=0$ and. $E^{\mu-1} \neq 0$ Several methods to calculate those matrices can be found in [19] and in [36]. The solution sequence is given for any $t \in \mathbf{Z}_{0+}[19]:$

$$
z_{t}=\Phi_{t} E z_{0}+\sum_{k=0}^{t+\mu-1} \Phi_{t-k-1} B_{c} c_{k}
$$

If, in addition, $A=A^{0}+K$ is non-singular then $\Phi_{t-1}=\Phi_{t} E A^{-1}$ and

$$
\Phi_{t-k-1}=\Phi_{t-k}\left(E A^{-1}\right)^{1}=\Phi_{t-k+1}\left(E A^{-1}\right)^{2}=\Phi_{t}\left(E A^{-1}\right)^{k+1}
$$

Then, the above expression (64) for the solution can be rewritten as follows:

$$
\begin{gathered}
z_{t}=\Phi_{t}\left(E z_{0}+\sum_{k=0}^{t+\mu-1}\left(E A^{-1}\right)^{k+1} B_{c} \mathcal{c}_{k}\right)=\Phi_{t} E\left(z_{0}+\sum_{k=0}^{t+\mu-1} E^{k} A^{-(k+1)} B_{\mathcal{c}} \mathcal{c}_{k}\right) \\
=\Phi_{t-1}\left(A z_{0}+\sum_{k=0}^{t+\mu-1}\left(E A^{-1}\right)^{k} B_{c} \mathcal{c}_{k}\right)
\end{gathered}
$$

The following result is concerned with the non-negativity of the solutions of the state-space descriptions (58) and (50):

Theorem 6. (non-negativity of the solution of the singular regular system). Assume that $(E, A)$ is regular. Then, the following properties hold for any given $L \geq n$ :

(i) If $A_{1} \succ 0, B_{1} \succ 0, N \succ 0$ and $\left(-B_{2}\right) \succ 0, z_{10} \succeq 0, z_{2 L} \succeq 0$ and $\left\{u_{t}\right\}_{0}^{\infty} \subset \boldsymbol{R}_{0+}$ in the state-space description (58)-(60) then $\left\{z_{1 t}\right\}_{0}^{\infty} \subset \boldsymbol{R}_{0+}$ and $\left\{z_{2 t}\right\}_{0}^{\infty} \subset \boldsymbol{R}_{0+}$. If, in addition, 
$z_{10} \succ 0$ and $z_{2 L} \succ 0$, or if $u_{0} \succ 0$, then $\left\{z_{1 t}\right\}_{0}^{\infty} \subset \boldsymbol{R}_{+}$and $\left\{z_{2 t}\right\}_{0}^{\infty} \subset \boldsymbol{R}_{+}$. If $\mu$ is the nilpotency index of $N$ and $t \leq L-\mu$ then the boundary condition $z_{2 L}$ can be removed and the given properties still hold on $[0, L]$, i.e., $\left\{z_{1 t}\right\}_{0}^{L} \subset \boldsymbol{R}_{+}$and $\left\{z_{2 t}\right\}_{0}^{L} \subset \boldsymbol{R}_{+}$.

(ii) Consider the state space description (50). Assume that $E \succ 0, K_{c} \succ I_{n}$ (i.e., $B_{c} \succ 0$ ), $\Phi_{i} \succ 0 ; i=-\mu,-\mu+1, \cdots, 0,1$, and that either $z_{0} \succ 0$ and $\left\{u_{t}\right\}_{0}^{\infty} \subset \boldsymbol{R}_{0+}$ or $z_{0} \succeq 0$ and $\left\{c_{t}\right\}_{0}^{\infty} \subset \boldsymbol{R}_{0+}$ with $c_{1} \succ 0$. Then, $\left\{z_{t}\right\}_{0}^{\infty} \subset \boldsymbol{R}_{+}$. If $z_{0} \succeq 0$ and $\left\{c_{t}\right\}_{0}^{\infty} \subset \boldsymbol{R}_{0+}$ then $\left\{z_{t}\right\}_{0}^{\infty} \subset \boldsymbol{R}_{0+}$.

(iii) Consider the state space description (50). Assume that $e_{k}^{T} \Phi_{1} E e_{j} \geq 0$ and $e_{k}^{T} \Phi_{i} B_{c} e_{j} \geq 0$; $\forall k, j \in \bar{n}$ ( $e_{k}$ being the $k$-th vector of the canonical basis); $i=-\mu,-\mu+1, \cdots, 0,1$. If $z_{0} \succeq 0$ and $\left\{c_{t}\right\}_{0}^{\infty} \subset \boldsymbol{R}_{0+}$ then $\left\{z_{t}\right\}_{0}^{\infty} \subset \boldsymbol{R}_{0+}$.

(iv) Consider the state space description (50). Assume that $A^{0} \succ-K$ (that is, $A=A^{0}+K \succ 0$ ) and $A=A^{0}+K$ is either monomial or a positive definite $M$-matrix (then non-singular with $A^{-1} \succ 0$ in both cases) and assume also that $E \succ 0, K_{c} \succ I_{n}, \Phi_{0} \succ 0$ and $\left\{c_{t}\right\}_{0}^{\infty} \subset \boldsymbol{R}_{0+}$ or $z_{0} \succeq 0$ and $\left\{c_{t}\right\}_{0}^{\infty} \subset \boldsymbol{R}_{0+}$ with $c_{1} \succ 0$. Then, $\left\{z_{t}\right\}_{0}^{\infty} \subset \boldsymbol{R}_{+}$.

(v) Consider the state space description (50). Assume that $A=A^{0}+K \succ 0$ is either monomial or a positive definite $M$-matrix (then non-singular with $A^{-1} \succ 0$ ). Assume also that $e_{k}^{T} \Phi_{1} E e_{j} \geq 0$ and $e_{k}^{T} \Phi_{i} B_{c} e_{j} \geq 0 ; \forall k, j \in \bar{n} ; i=-\mu,-\mu+1, \cdots, 0,1$. If $z_{0} \succeq 0$ and $\left\{c_{t}\right\}_{0}^{\infty} \subset \boldsymbol{R}_{0+}$ then $\left\{z_{t}\right\}_{0}^{\infty} \subset \boldsymbol{R}_{0+}$.

Proof. Property (i) is a direct consequence of (61) and (62).

To prove Property (ii), we rewrite (64) by calculating $x_{t}$ for any $t \in Z_{+}$from its previous sampled value $x_{t-1}$ as follows:

$$
z_{t}=\Phi_{1} E z_{t-1}+\sum_{k=t-1}^{t+\mu-1} \Phi_{t-k-1} B_{c} c_{k}
$$

equivalently,

$$
z_{t}=\Phi_{0}\left(A^{0}+K\right) z_{t-1}+\sum_{k=t-1}^{t+\mu-1} \Phi_{t-k-1} B_{c} c_{k}
$$

It follows from (68) and recursion to calculate the solution sequence that $E \succ 0$, $B_{c} \succ 0, \Phi_{i} \succ 0 ; i=-\mu,-\mu+1, \cdots, 0,1$; and either $z_{0} \succ 0$ and $\left\{c_{t}\right\}_{0}^{\infty} \subset \boldsymbol{R}_{0+}$ or $z_{0} \succeq 0$ and $\left\{c_{t}\right\}_{0}^{\infty} \subset \boldsymbol{R}_{0+}$ with $c_{1} \succ 0$ guarantee that $\left\{z_{t}\right\}_{0}^{\infty} \subset \boldsymbol{R}_{+}$. Additionally, if $z_{0} \succeq 0$ and $\left\{c_{t}\right\}_{0}^{\infty} \subset \boldsymbol{R}_{0+}$, then $\left\{z_{t}\right\}_{0}^{\infty} \subset \boldsymbol{R}_{0+}$.

The proof of Property (iii) follows similarly to that of Property (ii) under the weaker sufficiency-type conditions that $e_{k}^{T} \Phi_{1} E e_{j} \geq 0$ and $e_{k}^{T} \Phi_{i} B_{c} e_{j} \geq 0 ; \forall k, j \in \bar{n} ; i=-\mu,-\mu+$ $1, \cdots, 0,1$ which guarantee from (68) that, if $z_{0} \succeq 0$ and $\left\{c_{t}\right\}_{0}^{\infty} \subset \boldsymbol{R}_{0+}$, then $\left\{z_{t}\right\}_{0}^{\infty} \subset \boldsymbol{R}_{0+}$.

The proof of Property (iv) follows under the guidelines of that of Property (ii) for the case when $A$ is positive non-singular with positive inverse leading to (66), which can be rewritten with a finite memory in a similar way to (68). The proof of Property (v) follows from that of Property (iv) in a similar way as the proof of Property (iii) follows from that Property (ii).

Remark 7. The above result gives conditions for non-negativity of the solution for any nonnegative/positive initial conditions and non-negative/positive controls. However, the properties are not, in general, kept from canonical state-space representations to the initial state-space description and vice-versa.

The reachability of the singular regular version of the system is now discussed from the following extended version of (10), which is appropriate for such a purpose:

$$
E z_{t+1}=A^{0} z_{t}+G u_{t}+B^{0} c_{t}
$$


where, typically,

$$
G=\left[\begin{array}{c}
G_{x_{n \times n}} \\
0_{m \times n} \\
0_{o \times n}
\end{array}\right]
$$

if $\left\{u_{t}\right\}_{0}^{\infty}$ is used to a direct correcting control of the economic goods only based on feedback information on the preceding sample of some or all of them and eventually some or all of the reusable items. In the above model, $\left\{u_{t}\right\}_{0}^{\infty}$ is the correcting control which acts in such a way that only the economic goods variables can be directly driven by a control function, the other couplings from those variables to reusable and recycled ones being zero. Therefore, the potential reachability of (70) from the control sequence $\left\{u_{t}\right\}_{0}^{\infty}$ can hold only if the state variables of economic goods or product can be able to drive the remaining variables of the state vector for reachability purposes of a prescribed state vector.

The following corollary to Theorem 6 is direct to characterize the positivity of (70) and (73):

Corollary 3. Theorem 6 applies directly to (70) and (71), provided that $G_{x} \succ 0$ with the replacement $A \rightarrow A^{0}$ with $\mathrm{K}=0$.

The following reachability result holds for the singular regular system:

Theorem 7. (reachability of the singular regular system). Assume that $(E, A)$ is regular. Then, the following properties hold:

(i) The system (70) and (71) is n-step reachable from the correcting control sequence $\left\{u_{t}\right\}_{0}^{\infty}$ if and only if rank $R_{u n}=n+m+o$, where

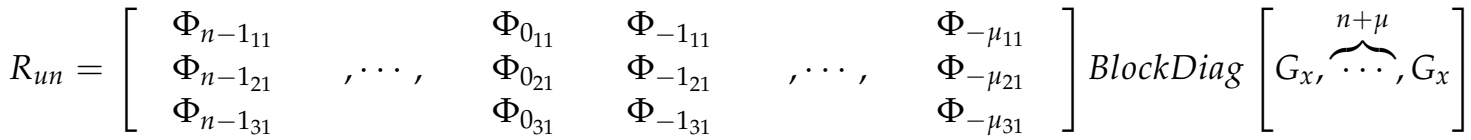

$$
\begin{aligned}
& \text { with } \\
& \Phi_{k}=\text { BlockMatrix }\left(\Phi_{k_{i j}} ; i, j \in \overline{3}\right)=\left[\begin{array}{lll}
\Phi_{k_{11}} & \Phi_{k_{12}} & \Phi_{k_{13}} \\
\Phi_{k_{21}} & \Phi_{k_{22}} & \Phi_{k_{23}} \\
\Phi_{k_{31}} & \Phi_{k_{32}} & \Phi_{k_{33}}
\end{array}\right] ; k=-\mu, \ldots,-1,0,1, \ldots, n-1 \\
& R_{c n 1}=R_{\Phi 1} \text { BlockDiag }[E_{c}^{0}, \overbrace{\cdots}^{n+\mu}, E_{c}^{0}] ; R_{c n 2}=R_{\Phi 2} \text { BlockDiag }[W_{c}, \overbrace{\cdots}^{n+\mu}, W_{c}]
\end{aligned}
$$

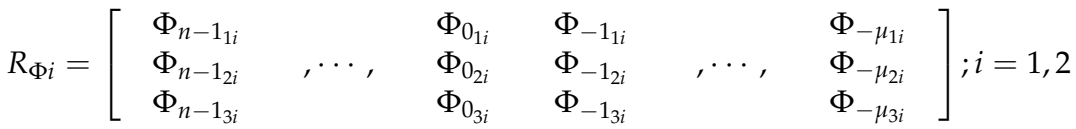

$$
\begin{aligned}
& \bar{R}_{u c n}=\left[R_{u n}, R_{c n}\right]=\left[R_{\Phi 1} \text { BlockDiag }[G_{x}, \overbrace{\cdots}^{n+\mu}, G_{x}], R_{\Phi 1} \text { BlockDiag }[E_{c}^{0}, \overbrace{\cdots}^{n+\mu}, E_{c}^{0}]+R_{\Phi 2} \text { BlockDiag }[W_{c}, \overbrace{\cdots}^{n+\mu}, W_{c}]\right]
\end{aligned}
$$


Proof. The reachability matrix $R_{u n}$ of the singular regular system (70) and (71) from the correcting control sequence under zero demand sequence is given by:

$$
R_{u n}=\left[\Phi_{n-1} G, \cdots, \Phi_{0} G, \Phi_{-1} G, \ldots, \Phi_{-\mu} G\right]
$$

Note that

$$
R_{u n}=\left[\Phi_{n-1}, \cdots, \Phi_{0}, \Phi_{-1}, \ldots, \Phi_{-\mu}\right] \text { BlockDiag }[G, \overbrace{\cdots}^{n+\mu}, G]
$$

and since $\Phi_{k} G=\left[\begin{array}{c}\Phi_{k_{11}} G_{x} \\ \Phi_{k_{21}} G_{x} \\ \Phi_{k_{31}} G_{x}\end{array}\right] ; k=-\mu, \ldots-1,0,1, \ldots, n-1$, then (77) becomes compacted as (72) and it has to be of full rank for reachability so that Property (i) is proved.

To prove Property (ii), note that the system (70) and (71) is $(n+m+o)$-step reachable from the demand sequence correcting control sequence $\left\{c_{t}\right\}_{0}^{\infty}$ if and only if $\operatorname{rank} R_{c n}=n+$ $m+o$, where $R_{c n}=R_{c n 1}+R_{c n 2}$ from (74) and (75) with $E_{c}^{0}$ and $W_{c}$ generating, respectively, $R_{c n 1}$ and $R_{c n 2}$ by inspection of (10) and (11) and following the arguments in the proof of Proposition (i).

To prove Property (iii), note that that the subsequent reachability matrix for the combined correcting control and demand should be full rank:

$$
R_{u c n}=\left[\Phi_{n-1}\left(G, B^{0}\right), \ldots, \Phi_{0}\left(G, B^{0}\right), \Phi_{-1}\left(G, B^{0}\right), \ldots, \Phi_{-\mu}\left(G, B^{0}\right)\right]
$$

Note also that $R_{c n 1}$ is similar to $R_{u n}$ [Property (i)] after performing the replacement $G_{x} \rightarrow E_{c}^{0}$, from inspection of (10)-(11). Note also that $R_{c n}=R_{c n 1}+R_{c n 2}$ is the reachability matrix associated with the demand sequence [Property (ii)]. Then, one gets from the combined Properties (i) and (ii) that the above reachability matrix (79) is similar to $\bar{R}_{u c n}$, defined in (76), which proves Property (iii).

Now consider the expression:

$$
\hat{z}_{t+n}=z_{t+n}+\varepsilon_{t+n}=z^{*}+\varepsilon_{t+n}=R_{n} \hat{u}_{t}=R_{n} R_{n}^{T} \sigma_{t}
$$

which is the reachability tracking objective which is any prefixed state $x^{*}$ at $t+n$ from the state at the $t$-sample plus the algebraic addition/subtraction of terms which do not depend on the particular active primary input $\hat{u}_{t}$ to be generated on $[t, t+n+m+o)$ which is a finite subsequence of either the correcting control, demand or mixed correcting control/demand (see Theorem 7 [(i)-(iii)]) and $\sigma_{t}$ are the values of an auxiliary input which generates $\hat{u}_{t}=R_{n}^{T} \sigma_{t}$ according to

$$
\sigma_{t}=\left(R_{n} R_{n}^{T}\right)^{-1} \hat{z}_{t+n}=\left(R_{n} R_{n}^{T}\right)^{-1} R_{n} \hat{u}_{t}
$$

provided that the reachability matrix is full rank, which implies that the $(n+m+o)$-square matrix $R_{n} R_{n}^{T}$ is nonsingular, and

$$
R_{n}=\left\{\begin{array}{l}
R_{u n} \text { ifthecontrol onthetimeinterval }[t, t+n+m+o) \text { is } \hat{u}_{t} \leftarrow\left\{u_{j}\right\}_{t}^{t+n-1} \text { (Theorem 7(i)) } \\
R_{c n} \text { ifthecontrolonthetimeinterval }[t, t+n+m+o) \text { is } \hat{u}_{t} \leftarrow\left\{c_{j}\right\}_{t}^{t+n-1} \text { (Theorem7 (ii)) } \\
R_{c n} \text { ifthecontrolonthetimeinterval }[t, t+n+m+o) \text { is } \hat{u}_{t} \leftarrow\left\{\left(\begin{array}{c}
u_{j} \\
c_{j}
\end{array}\right)\right\}_{t}^{t+n-1} \text { (Theorem7(iii)) }
\end{array}\right.
$$

The above comments together with Theorem 7 lead to the subsequent result: 
Theorem 8. Assume that $R_{n} \succ 0$ and that $R_{n} R_{n}^{T}$ is either monomial positive or a positive definite $M$ - matrix. Then, $\sigma_{t} \succ 0, \hat{u}_{t} \succ 0, u_{t} \succ 0$ for non-negative initial conditions at t provided that $x^{*} \succ 0$ is such that $\hat{z}_{t+n} \succ 0$.

Remark 8. Note that in order to discuss the positivity properties, the specific use of Theorem 7 is not necessary since the reachability matrices for one of the inputs or both of them in a combined operation can be positive without requiring for $\Phi_{i}$ and $G$ to be specifically positive, since only parametrical combinations by manipulating them build the various reachability matrices.

Remark 9. If $K_{c} \prec I_{n}$, which includes, in particular, the case when $K_{c}=0$ (namely, no correcting action is taken based on demand feedback information and $B=B^{0}=\left[\begin{array}{c}-I_{n} \\ W_{c} \\ 0_{o \times n}\end{array}\right]$ ) then Theorem 8 is still valid by the appropriate definition of the sequence $\left\{\varepsilon_{t}\right\}$ in (80), on the time interval of interest, to achieve the positivity of the claimed tracking objective. To figure out the above comment, note from (50) that one gets for the particular case when $W_{c}=0$ :

$$
E z_{t+1}+c_{t}=\left(A^{0}+K\right) z_{t}+K_{c} c_{t} \succeq\left(A^{0}+K\right) z_{t}
$$

Remark 10. Note that the computation of the extended control $\hat{u}_{t}$ (see (80)) on the time interval of interest gives a feedback information on control gain matrices, which can generalize the timeinvariant parameterization of the correcting control law (4).

A simple way to combine regularity and the typical stability concept of characteristic zeros lying in the open circle centered at zero of radius unity under small parametrical perturbations is directly as follows. Firstly, state that pair $(E, A)$ is said to be stable if $\operatorname{det}(z E-A) \neq 0$ in $|z| \geq 1$ and $\operatorname{det}(z E-A)$ is not identically zero in $|z|<1$. Equivalently, $Z(E, A) \neq \Phi$, i.e., the set of zeros $\operatorname{det}(z E-A)$ is nonempty, and $Z(E, A) \cap$ $\{z \in C:|z| \geq 1\}=\Phi$. Note that if $(E, A)$ is stable then it is trivially regular. However, note that, for stability purposes, the requirement that $\operatorname{det}(z E-A)$ is not identically zero in $|z|<1$ if $\operatorname{det}(z E-A) \neq 0$ in $|z| \geq 1$ is not needed for stability from a stable regular pair since, otherwise, then all solution is trivial and then stable. Thus, the condition $Z(E, A) \neq \Phi$ is unnecessary.

Assertion 1. (stability of a regular pair). Assume that $(E, A)$ is regular with $\operatorname{det}(z E-A) \neq 0$ for $|z| \geq 1$. Then, $(E, A)$ is stable irrespective of $\operatorname{det}(z E-A)$, being identically zero in $|z|<1$ or not.

Proof. Assume that $Z(E, A) \neq \Phi$ and $Z(E, A) \cap\{z \in C:|z| \geq 1\}=\Phi$. Then, $\operatorname{det}(z E-A)=0$ for points in a nonzero subset in the open unit circle centered at zero so that $(E, A)$ is stable.

Now assume that $Z(E, A)=\Phi$, which leads to $\operatorname{det}(z E-A) \neq 0$ for any $z \in C$. Then, $(z E-A) X(z)=0$ if and only if $X(z) \in \operatorname{Ker}(z E-A)=\{0\}$ for all $z \in C$, where $X(z)$ is the $z$-transform of the solution sequence $\left\{z_{t}\right\}_{0}^{\infty}$ of $E z_{t+1}=A z_{t}$ so that $\left\{z_{t}\right\}_{t=1}^{\infty} \equiv 0$ so that $(E, A)$ is stable.

The subsequent direct result relies on guaranteeing the stability of a perturbed pair $(E, A)$ under that of a nominal one $\left(E_{0}, A_{0}\right)$ and it can be easily reformulated in a dual context which is omitted.

Theorem 9. (stability of a disturbed regular pair). Assume that $\left(E_{0}, A\right)$ is stable and that $\sup _{\theta \in[0,2 \pi)}\left\|\left(e^{i \theta} E_{0}-A_{0}\right)^{-1}\left(e^{i \theta} \widetilde{E}-\widetilde{A}\right)\right\|<1$, where $\widetilde{E}=E-E_{0}$ and $\widetilde{A}=A-A_{0}$. Then, $(E, A)$ is stable. 
Proof. Note through direct calculations that

$$
z E-A=\left(z E_{0}-A_{0}\right)\left(I_{n}+\left(z E_{0}-A_{0}\right)^{-1}(z \widetilde{E}-\widetilde{A})\right) ; \forall z \notin Z\left(E_{0}, A_{0}\right)
$$

where $Z\left(\hat{E}_{0}, \hat{A}_{011}\right):=\left\{z \in C: \operatorname{det}\left(z E_{0}-A_{0}\right)=0\right\}$, so that $\left(z E_{0}-A_{0}\right)^{-1}$ exists in $|z| \geq 1$ since $\left(\hat{E}_{0}, \hat{A}_{0}\right)$ being stable implies that $\operatorname{det}\left(z E_{0}-A_{0}\right) \neq 0$ in $|z| \geq 1$ what implies in turn that $\left(E_{0}, A_{0}\right)$ is regular. Then,

$$
\operatorname{det}\left(z E_{0}-A_{0}\right)=\operatorname{det}\left(z E_{0}-A_{0}\right) \operatorname{det}\left(I_{n}+\left(z E_{0}-A_{0}\right)^{-1}(z \widetilde{E}-\widetilde{A})\right) \neq 0 ; \forall z \notin Z\left(E_{0}, A_{0}\right)
$$

provided that $\|G\|_{\infty}=\sup _{\theta \in[0,2 \pi)}\left\|\left(e^{i \theta} E_{0}-A_{0}\right)^{-1}\left(e^{i \theta} \widetilde{E}-\widetilde{A}\right)\right\|<1$, where $i=\sqrt{-1}$ is the complex unit and $\|G\|_{\infty}$ is the $H_{\infty}$-norm of $\left(z E_{0}-A_{0}\right)^{-1}(z \widetilde{E}-\widetilde{A})$, under the given assumption that $\left(E_{0}, A_{0}\right)$ is stable.

\section{Numerical Examples}

This section contains some numerical simulation examples, with the purpose of illustrating the reachability issues and theoretical properties of the Leontief-type model discussed previously.

Example 1. Consider the system (1)-(4) parameterized by the following matrices:

$$
\begin{gathered}
A_{x}=\left(\begin{array}{lll}
0.6 & 0.3 & 0.2 \\
0.4 & 0.2 & 0.3 \\
0.2 & 0.3 & 0.5
\end{array}\right), A_{y}=\left(\begin{array}{cc}
0.3 & 0 \\
0 & 0.2 \\
0.1 & 0.05
\end{array}\right), B_{x}=\left(\begin{array}{ccc}
0.73 & 0.3 & 0.4 \\
0.5 & 0.4 & 0.6 \\
0.27 & 0.36 & 0.5
\end{array}\right), B_{y}=\left(\begin{array}{cc}
0.1 & 0.05 \\
0.05 & 0.1 \\
0.01 & 0.05
\end{array}\right) \\
W_{x}=\left(\begin{array}{lll}
0.1 & 0.1 & 0.05 \\
0.1 & 0.1 & 0.04
\end{array}\right), W_{y}=\left(\begin{array}{cc}
0.95 & 0 \\
0 & 0.99
\end{array}\right), W_{c}=\left(\begin{array}{ccc}
0.2 & 0.05 & 0.01 \\
0.01 & 0.2 & 0.35
\end{array}\right) \\
D_{x}=\left(\begin{array}{ccc}
0.6 & 0.2 & 0.05 \\
0.1 & 0.1 & 0.04
\end{array}\right), P=2 A_{y}
\end{gathered}
$$

Notice that all the above matrices are positive, hence fulfilling Assumption 1. Moreover, it can be readily verified that

$$
\bar{C}_{x}^{0}=\left(\begin{array}{ccc}
2.43 & 1.3 & 0.8 \\
3.2 & 4.3 & 1.55 \\
1.29 & 1.28 & 1.51
\end{array}\right) \succ 0
$$

and

$$
\bar{E}_{c}^{0}-\bar{C}_{x}^{0} \bar{B}_{x}^{-1} B_{y}\left(I_{m}-W_{y}\right)^{-1} W_{c}=\left(\begin{array}{ccc}
-0.309 & 0.036 & -0.207 \\
0.847 & -6.531 & -10.121 \\
0.229 & 2.277 & 2.939
\end{array}\right) \nsucc 0
$$

while $I_{m}-W_{y}=\operatorname{diag}(0.05,0.01)$ is monomial. The matrix

$$
\bar{B}_{x}=\left(\begin{array}{ccc}
1.43 & 1 & 0.7 \\
1.6 & 1.5 & 1.05 \\
0.79 & 0.88 & 0.71
\end{array}\right) \succ 0
$$

is positive but it is not monomial nor a Z-matrix. In addition, $R_{c}$ is full rank but is not even positive, monomial, nor a Z-matrix, with $n=3$ :

$$
R_{c}=10^{4}\left(\begin{array}{ccccccccc}
-0.0002 & 0.0012 & 0.0018 & -0.0031 & 0.0284 & 0.0419 & 0.0288 & -0.1786 & -0.5486 \\
0.0008 & -0.0076 & -0.0112 & 0.0170 & -0.1751 & -0.2573 & -0.1771 & 1.0761 & 3.3322 \\
-0.0007 & 0.0084 & 0.0123 & -0.0180 & 0.1917 & 0.2815 & 0.1940 & -1.1716 & -3.6354
\end{array}\right)
$$


These conditions only allow guaranteeing some of the items (1) and (3) of Theorem 1. The vector of demands, however, may still be calculated by (24). If we consider the target vector as $x *=\left[\begin{array}{lll}600 & 4600 & 4900\end{array}\right]$ and the boundary demand vector is $c_{t+n}=$ $\left[\begin{array}{lll}1 & 1 & 1\end{array}\right]$ with initial conditions $x_{0}=\left[\begin{array}{lll}5 & 46 & 11\end{array}\right]$, the application of (24) to (14) generates the trajectory depicted in Figure 1. It is observed in Figure 1 that the control law is able to drive the state to the target one. However, since conditions (2), (4), and (5) of Theorem 1 are not satisfied, some of the states are negative at some iteration as shown in Figure 1. Furthermore, the Figure 2 displays the demands vector components. It can be seen in this Figure that some of the demands are negative at some iterations, due to the fact that some conditions of the Theorem 1 are not satisfied.

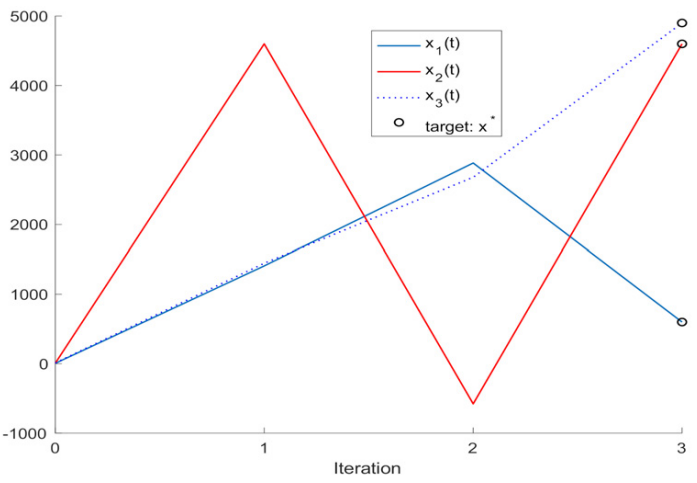

Figure 1. Evolution of stock vector $x_{t}$ under the control law (24) in Example 1.
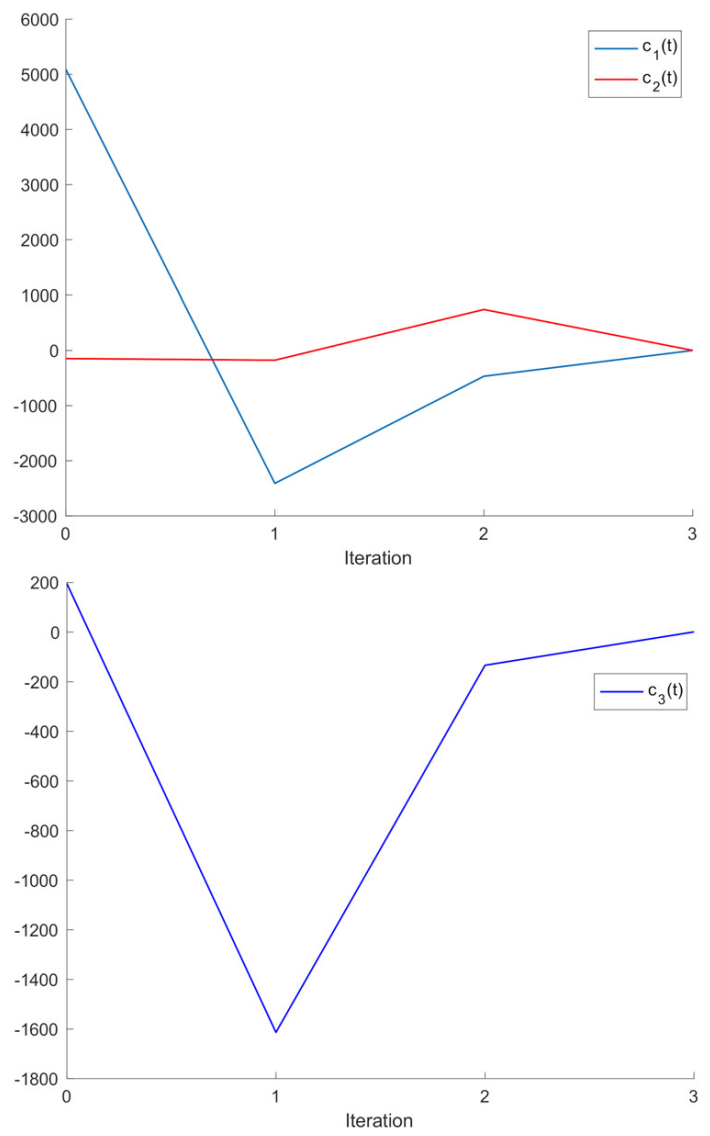

Figure 2. Demands vector components in Example 1. 
Example 2. Consider now the system (1)-(4) parameterized by the matrices:

$$
\begin{gathered}
A_{x}=\operatorname{diag}(0.6,0.2,0.3), A_{y}=\left(\begin{array}{cc}
0.3 & 0 \\
0 & 0.2 \\
0.1 & 0.05
\end{array}\right), B_{x}=\operatorname{diag}(0.7,0.4,0.5), \\
B_{y}=\left(\begin{array}{cc}
0.1 & 0 \\
0 & 0.1 \\
0 & 0
\end{array}\right) \\
W_{x}=\left(\begin{array}{ccc}
0.1 & 0 & 0 \\
0 & 0.1 & 0
\end{array}\right), W_{y}=\left(\begin{array}{cc}
0.95 & 0 \\
0 & 0.99
\end{array}\right), W_{c}=\left(\begin{array}{ccc}
0.2 & 0.05 & 0.01 \\
0.01 & 0.2 & 0.35
\end{array}\right) \\
D_{x}=\left(\begin{array}{ccc}
0.6 & 0.2 & 0.05 \\
0.01 & 0.01 & 0.3
\end{array}\right), P=6 A_{y}
\end{gathered}
$$

while the target and initial conditions are the same as in Example 1. Now we have

$$
\bar{C}_{x}^{0}=\left(\begin{array}{ccc}
4.3 & 0 & 0 \\
0 & 12.2 & 0 \\
1 & 2.5 & 1.2
\end{array}\right) \succ 0
$$

and

$$
\bar{E}_{c}^{0}-\bar{C}_{x}^{0} \bar{B}_{x}^{-1} B_{y}\left(I_{m}-W_{y}\right)^{-1} W_{c}=\left(\begin{array}{ccc}
3.489 & 1.122 & 0.224 \\
0.229 & 3.571 & 8 \\
1.627 & 1.817 & 1.578
\end{array}\right) \succ 0
$$

The matrix $I_{m}-W_{y}=\operatorname{diag}(0.05,0.01)$ is monomial and $\bar{B}_{x}=\operatorname{diag}(0.9,1.4,0.5) \succ 0$ is positive and monomial. However, $R_{c}$ is full rank but does not have a positive inverse $\left(R_{c} R_{c}^{T}\right)^{-1}$. The evolution of the stock vector is displayed in Figure 3. It is observed that the stock vector remains positive at all time but the demands components, displayed in Figure 4, have some negative components. Therefore, it can be concluded that condition (4) of Theorem 1 is necessary to ensure the nonnegativity of the stock vector but does not guarantee the positivity of the demands.

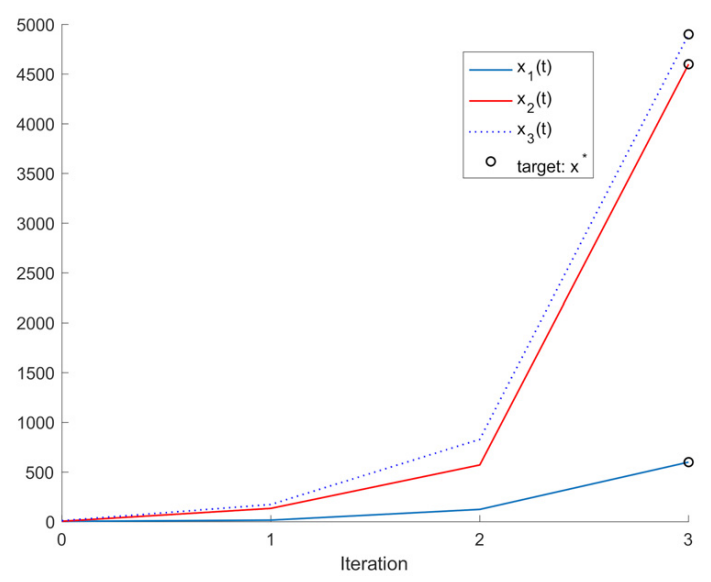

Figure 3. Evolution of stock vector $x_{t}$ in Example 2. 

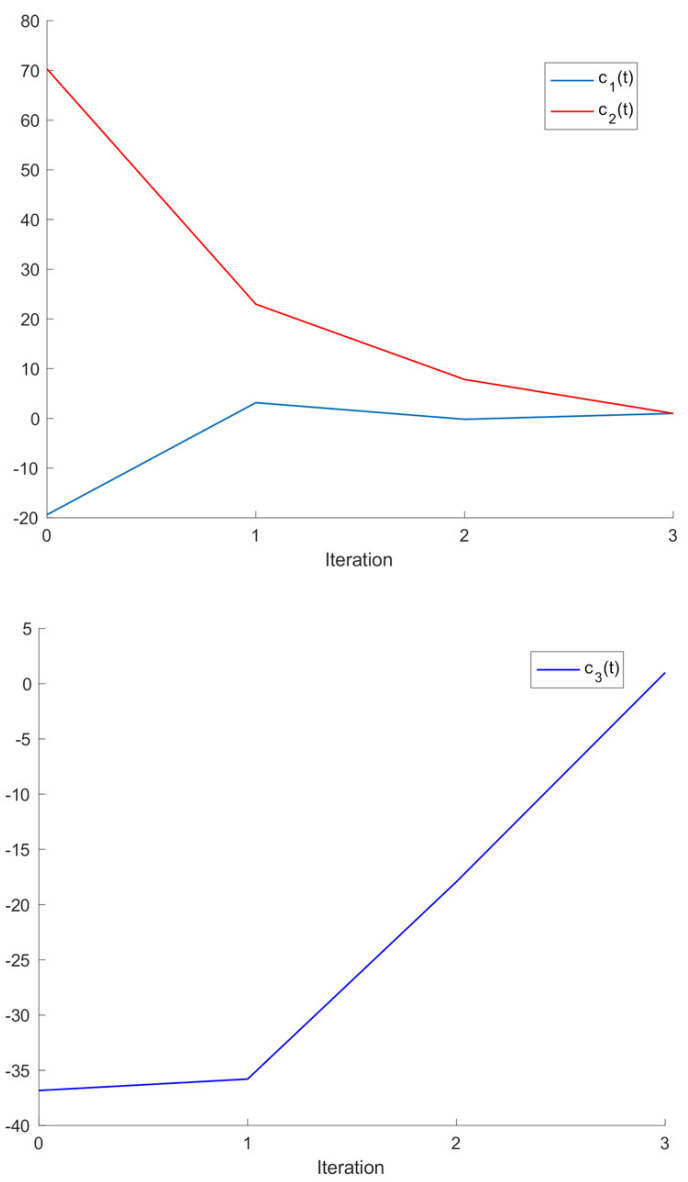

Figure 4. Demands vector in Example 2.

Example 3. Consider now the system (1)-(4) parameterized by the matrices:

$$
\begin{gathered}
A_{x}=\operatorname{diag}(0.6,0.2,0.3), A_{y}=\left(\begin{array}{cc}
0.3 & 0 \\
0 & 0.2 \\
0.1 & 0.05
\end{array}\right), B_{x}=\operatorname{diag}(0.7,0.4,0.5), \\
B_{y}=\operatorname{diag}(0.1,0.1 ; 0.1) \\
W_{x}=\left(\begin{array}{ccc}
0.1 & 0 & 0 \\
0 & 0.1 & 0
\end{array}\right), W_{y}=\operatorname{diag}(0.95,0.99,0.94), W_{c}=\operatorname{diag}(0.2,0.2,0.93) \\
D_{x}=\operatorname{diag}(0.6,0.01,0.15), P=6 A_{y}
\end{gathered}
$$

while the target and initial conditions are the same as in Example 1. Now we have:

$$
\begin{gathered}
\bar{C}_{x}^{0}=\operatorname{diag}(4.3,12.2,1.491) \succ 0, \\
\bar{E}_{c}^{0}-\bar{C}_{x}^{0} \bar{B}_{x}^{-1} B_{y}\left(I_{m}-W_{y}\right)^{-1} W_{c}=\operatorname{diag}(3.489,3.571,0.461) \succ 0
\end{gathered}
$$

while $I_{m}-W_{y}=\operatorname{diag}(0.05,0.01,0.06)$ is monomial and $\bar{B}_{x}=\operatorname{diag}(0.9,1.4,0.5833) \succ 0$ is positive and monomial. Moreover, $R_{c}$ is full rank and now possesses a positive inverse $\left(R_{c} R_{c}^{T}\right)^{-1}=10^{-3} \operatorname{diag}(0.0523,0.0008,0.4057)$. The evolution of the stock vector is displayed in Figure 5. It is observed that the stock vector remains positive at all time and, in addition, the demands components, displayed in Figure 6, are all of them positive as well. Therefore, it can be concluded that both conditions (4) and (5) of Theorem 1 are necessary to ensure the nonnegativity of the stock and demands vectors in order to obtain a model with full physical meaning. It is also remarkable to comment that in this case $(n=3)$, the examples we are using are the minimum number of samples needed to achieve the controllability of 
the system, as commented in Corollary 1 . More samples could be used if the stocks vector is to be fixed at a different larger iteration time.

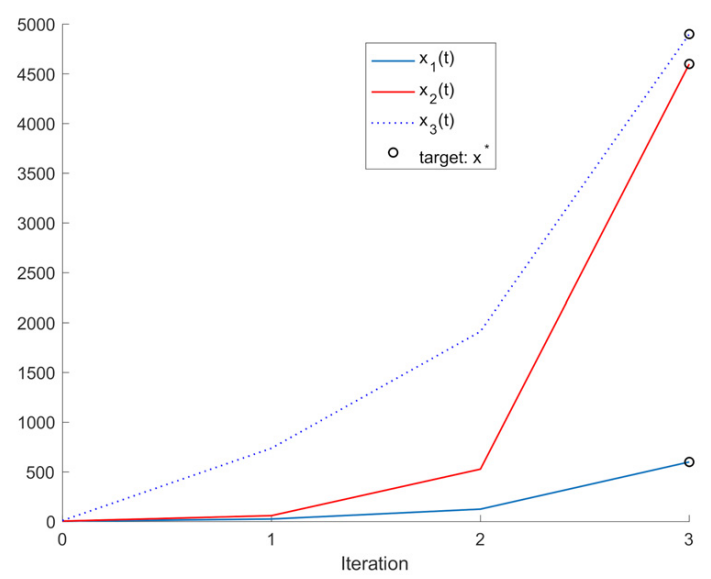

Figure 5. Evolution of stocks vector $x_{t}$ in Example 3.
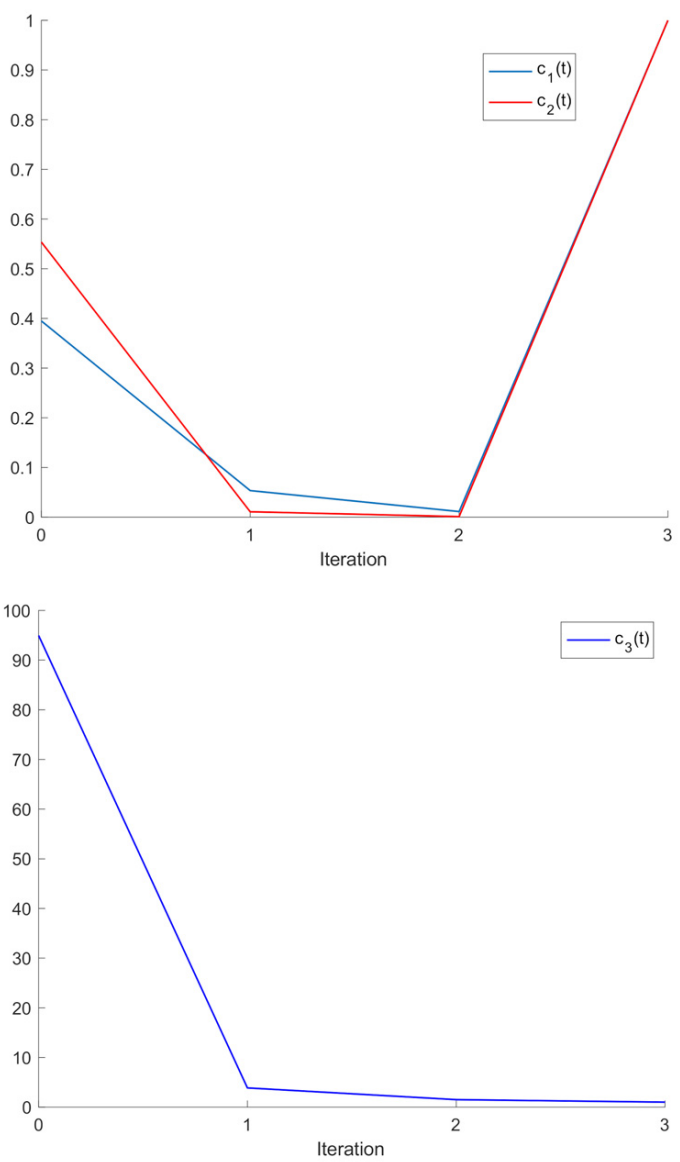

Figure 6. Demands vector in Example 3.

Example 4. Consider now the system (1)-(4) parameterized by the matrices:

$$
\begin{gathered}
A_{x}=\operatorname{diag}(0.6,0.2,0.3), A_{y}=\operatorname{diag}(0.3,0.2,0.3), B_{x}=\operatorname{diag}(0.7,0.4,0.5), \\
B_{y}=\operatorname{diag}(0.1,0.1 ; 0.1) \\
W_{x}=\operatorname{diag}(0.1,0.1,0.05), W_{y}=\operatorname{diag}(0.95,0.99,0.94), W_{c}=\operatorname{diag}(0.2,0.2,0.93) \\
D_{x}=\operatorname{diag}(0.6,0.01,0.15), P=6 A_{y}
\end{gathered}
$$


The control matrices are given by $K_{y}=0, K_{c}=0.95 I_{3}$ and the remaining parameters are the same as in Example 1. The matrix $K_{x}$ is selected in order to place the poles of the closed-loop system at $\{0.2,0.4,0.6\}$ so that the characteristic polynomial (45) takes the form $z^{3}-1.2029 z^{2}+0.4417 z-0.0482$. This polynomial defines the values of $\alpha_{i}$ in Equation (44) according to Theorem 2 . The problem of pole placement by state feedback is well-known in the control theory literature and its solution can be calculated by means of several available algorithms [28]. The solution to this case is $K_{x}=\operatorname{diag}(-4.12,-11.64,-1.14)$. The $c_{t}$ vector is now fixed to [1 111 ] at all iteration. The Figure 7 displays the evolution of the stock vector, while the Figure 8 displays the value of the feedback control signal $u_{t}$. It is observed in Figure 7 that the stock vector converges to an equilibrium point due to the action of the feedback control law. This is the effect of the considered control, the stabilization of an otherwise unstable system, as Figure 9 shows for the uncontrolled case. Moreover, since it is seen in Figure 7 that the stock vector converges to an equilibrium point, we can verify that Proposition 2 holds in this example because $\left(\bar{B}_{x}-\bar{C}_{x}\right) x=\left(\bar{E}_{c}-B_{y}\left(I_{3}-W_{y}\right)^{-1} W_{c}\right) c=$ $\left[\begin{array}{lll}5 & 19 & 2.875\end{array}\right]$.

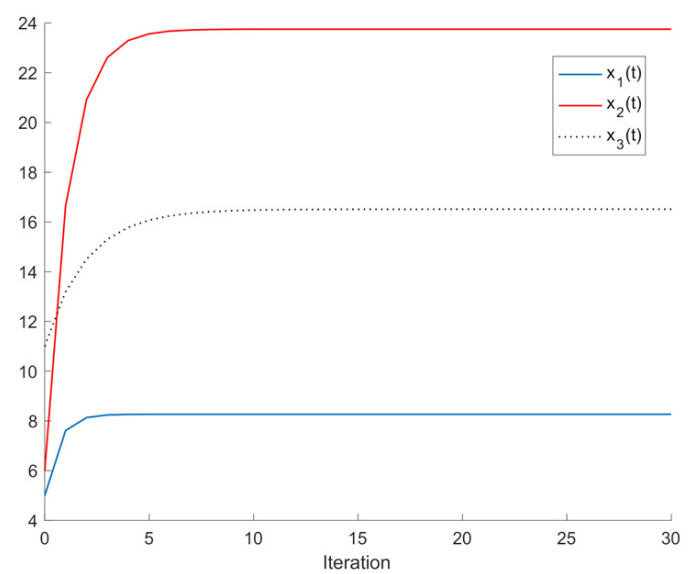

Figure 7. Evolution of the stock vector $x_{t}$ in Example 4.

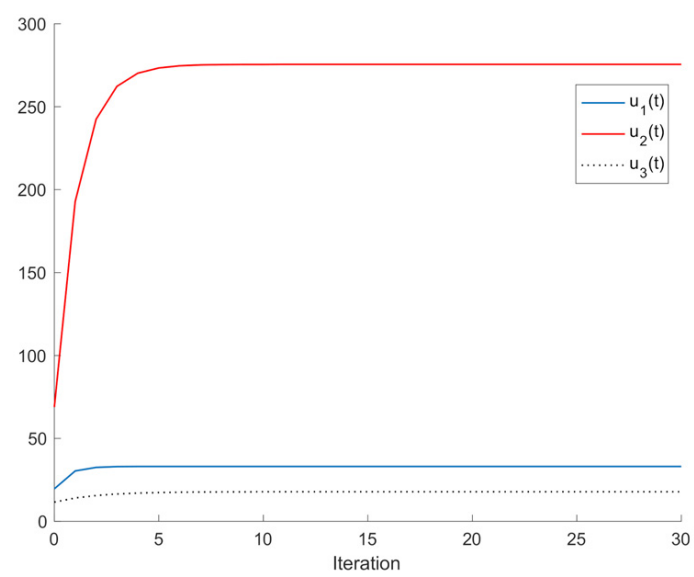

Figure 8. Feedback control signal in Example 4. 

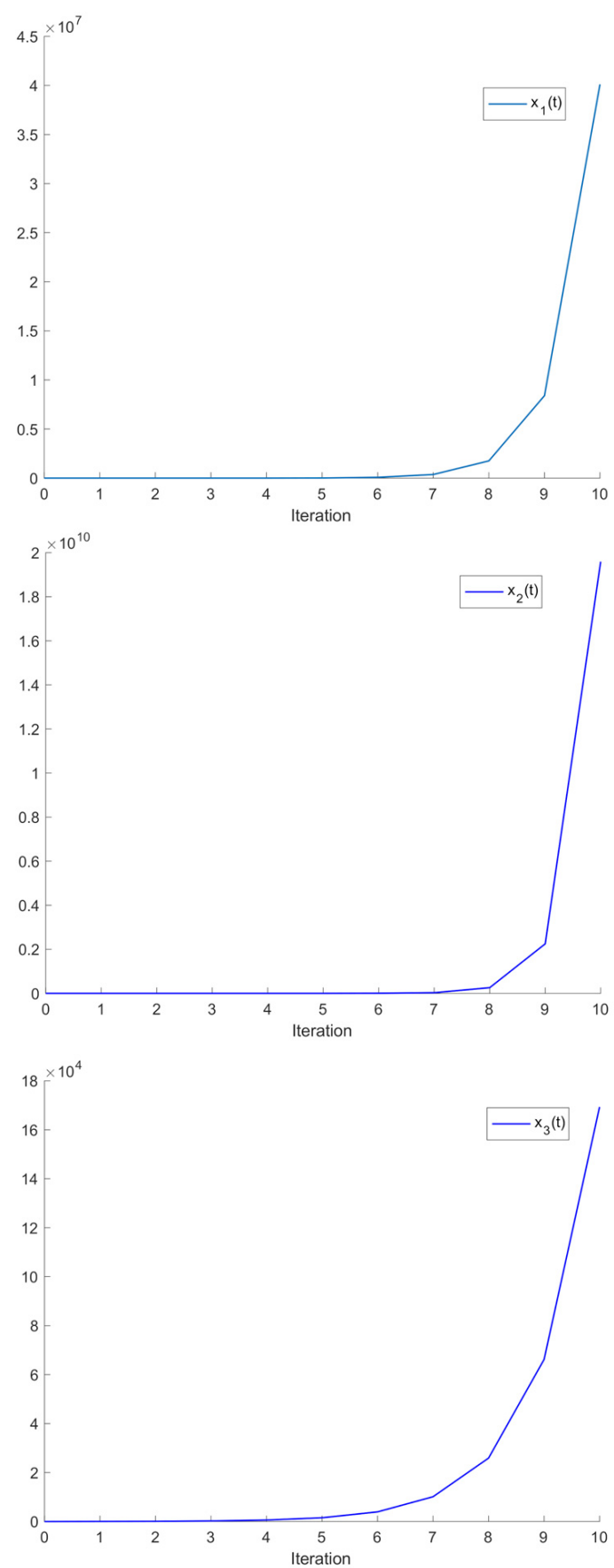

Figure 9. Evolution of the stock vector $x_{t}$ for the uncontrolled case in Example 4 .

Example 5. Consider now the system (1)-(4) parameterized by the same matrices as in Example 4. The control matrix, $K_{x}$, is now expressed in its dyadic form, $b \hat{k}_{x}^{T}$ where $b$ is given by [1 2 2 3$]$. The reachability matrix is given by:

$$
R_{c b}=\left(\begin{array}{ccc}
1.111 & 5.308 & 25.365 \\
1.428 & 12.449 & 108.484 \\
5.1429 & 13.1510 & 33.629
\end{array}\right)
$$

whose determinant is 439.37 . Therefore, the matrix is full rank and the system is reachable, but the positivity of the economic goods is not guaranteed at the first samples since the non-singular reachability matrix is neither monomial positive (see, for instance, Theorem 
2) nor a non-singular M- matrix. The control vector is calculated by (36) so as to obtain the same closed loop polynomial, as considered in Example 4. The solution is:

$$
\hat{k}_{x}^{T}=\left[\begin{array}{c}
8.62 \\
-16.59 \\
-0.14
\end{array}\right]
$$

The evolution of the stock vector is depicted in Figure 10, while Figure 11 displays the control signal. It is observed in Figure 10 that the stock vector reaches an equilibrium point. However, it can readily been verified that (48) from Theorem 3 holds but (49) does not. Consequently, the solutions are not nonnegative at all time, despite being asymptotically. Thus, Theorem 3 can be used to check the conditions to guarantee the nonnegativity of the solutions. It is to be pointed out that the control vector is selected to achieve a desired closed-loop characteristic polynomial. Therefore, once the closed-loop dynamics are selected it is difficult to guarantee the positivity of the solutions.

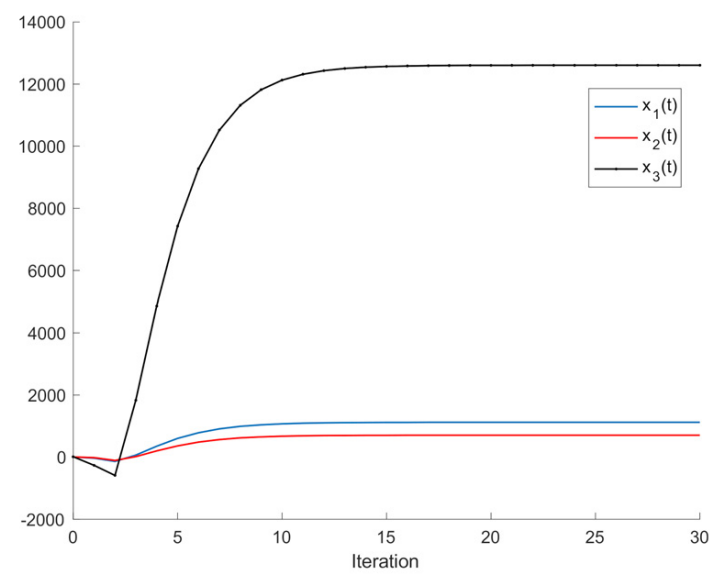

Figure 10. Evolution of the stock vector $x_{t}$ in Example 5 under a dyadic $K_{x}=b \hat{k}_{x}^{T}$ control matrix.

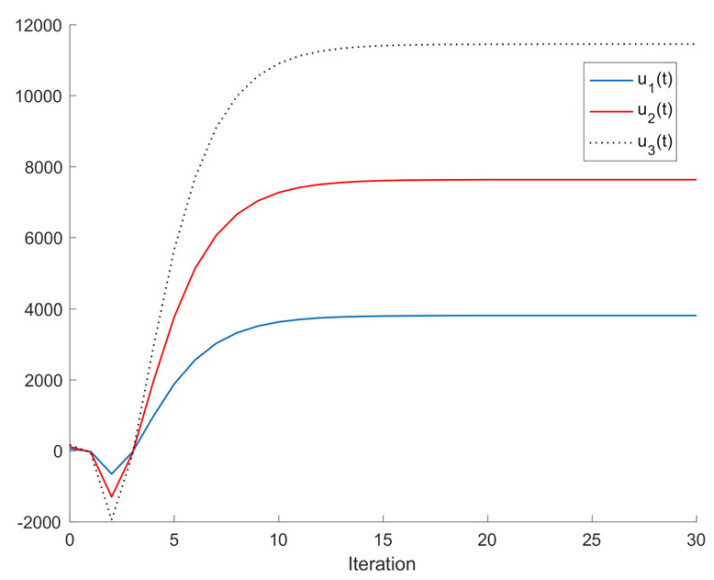

Figure 11. Values of the control signal $u_{t}$ in Example 5.

Example 6. Consider the system (1)-(4) with the matrices:

$$
\begin{gathered}
A_{x}=\operatorname{diag}(0.6,0.2,0.3), A_{y}=\operatorname{diag}(0.3,0.2,0.05), B_{x}=\operatorname{diag}(0.7,0.4,0.5), \\
B_{y}=\operatorname{diag}(0.1,0.1 ; 0.1) \\
W_{x}=\operatorname{diag}(0.1,0.1,0.05), W_{y}=\left(\begin{array}{ccc}
0.95 & 0 & 0 \\
0 & 0.99 & 0 \\
0 & 0.8 & 0
\end{array}\right), W_{c}=\operatorname{diag}(0.2,0.2,0.93) \\
D_{x}=\operatorname{diag}(0.6,0.01,0.15), P=6 A_{y}
\end{gathered}
$$


The control matrices are considered to be $K_{x}=0.015 I_{3}, K_{y}=0.02 I_{3}$, and $K_{c}=0.09 I_{3}$. This example is devoted to show the conditions under which the regularity in the absence of control is preserved when a control action is added and vice-versa, as discussed in Corollary 2. Therefore, Figures 12 and 13 show the 2-norm of the $G(k)$ matrices in relation to the norm of error matrices $\widetilde{G}(k)$. It is observed that the norm of the error matrices is much less than the norm of the uncontrolled and controlled ones. Since the error norm is sufficiently small compared to the norm of the original matrices (uncontrolled and controlled ones), the regularity of one implies the regularity of the other. In fact, it can be readily checked that both systems are regular since $\operatorname{det}(\lambda E-A)=10.24$ for a value of $\lambda=10$ and $\operatorname{det}\left(\lambda E-A_{0}\right)=11.81$. The constants $\varepsilon_{\widetilde{G}}(k)$ appearing in Corollary 2 are given for the first 4 values of $k$ by [0.0037 0.00040 .00030 .0003$]$. These values are pretty conservative since they are too low. Therefore, the definition of a more accurate bounding for the Corollary 2 constants is an open problem.

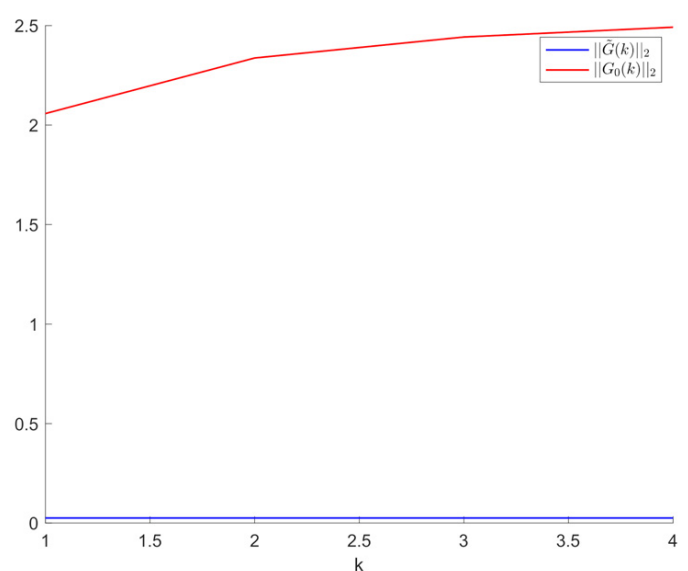

Figure 12. Relation between the 2-norm of the uncontrolled matrix $G_{0}(k)$ and error matrix $\widetilde{G}(k)$ in Example 6.

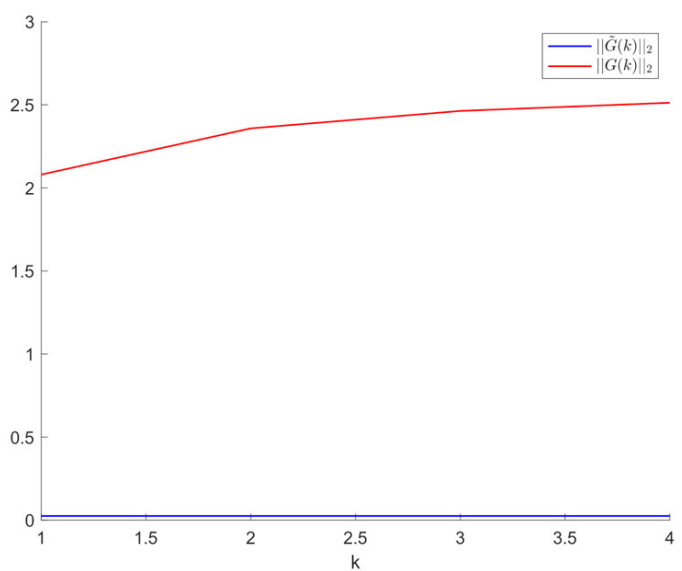

Figure 13. Relation between the 2-norm of the controlled matrix $G(k)$ and error matrix $\widetilde{G}(k)$ in Example 6.

Example 7. This last example is concerned with the stability of a perturbed descriptor system provided the stability of the unperturbed one. Thus, an illustrative example of Assertion 1 and Theorem 9 will be provided now. To this end, consider the descriptor system given by:

$$
E_{0}=\left(\begin{array}{ll}
1 & 0 \\
0 & 0
\end{array}\right), A_{0}=\left(\begin{array}{ll}
0.1 & 0.2 \\
0.3 & 0.4
\end{array}\right), B=\left[\begin{array}{l}
0 \\
1
\end{array}\right], C=\left[\begin{array}{ll}
1 & 0
\end{array}\right], \mathrm{D}=0
$$


This system has an economic good variable, a reusable recycling good variable, and no non-renewable product. This system is regular since for $\lambda=10, \operatorname{det}\left(\lambda E_{0}-A_{0}\right)=-4.02 \neq$ 0 . Moreover, $\operatorname{det}\left(z E_{0}-A_{0}\right)=-0.4 z-0.02$ with a single root at $z=-0.05$. Thus, according to Assertion 1 the regular system $\left(E_{0}, A_{0}\right)$ is stable since $\operatorname{det}\left(z E_{0}-A_{0}\right) \neq 0$ for $|z| \geq 1$. This fact can be verified in Figure 14, where the output of the system for the sinusoidal input $u=2 \sin (2 \pi 0.3 t)$ is depicted. As can be observed in Figure 14, the output remains bounded for all time, being the system input-output stable.

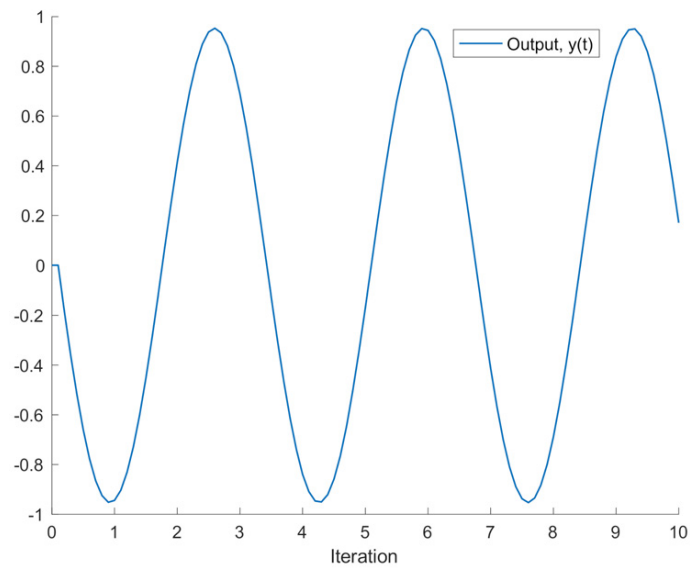

Figure 14. Output of the stable singular system $\left(E_{0}, A_{0}\right)$ in Example 7.

Now, the system is perturbed to

$$
E=\left(\begin{array}{cc}
0.9 & 0 \\
0 & 0
\end{array}\right), A=1.12 \cdot\left(\begin{array}{cc}
0.1 & 0.2 \\
0.3 & 0.4
\end{array}\right)
$$

and the question to answer is whether the system is still stable or not. In order to clarify this point, Theorem 9 can be employed. Thus, Figure 15 shows the value of $\sup _{\theta \in[0,2 \pi)}\left\|\left(e^{i \theta} E_{0}-A_{0}\right)^{-1}\left(e^{i \theta} \widetilde{E}-\widetilde{A}\right)\right\|$ with respect to $\theta$ and its limit of unity. It is seen in Figure 15 that the perturbed system $(E, A)$ has the value of $\sup _{\theta \in[0,2 \pi)}\left\|\left(e^{i \theta} E_{0}-A_{0}\right)^{-1}\left(e^{i \theta} \widetilde{E}-\widetilde{A}\right)\right\|$ below unity for all value of $\theta$. Accordingly, the perturbed system $(E, A)$ is stable. This fact can be verified by Figure 16 where the outputs of the unperturbed and perturbed systems are depicted. It is observed that both of them are bounded for all time. Additionally, $\operatorname{det}(z E-A)=-0.4032 z-0.0251$ whose root is $z=-0.062$ corresponding to a stable system from Assertion 1.

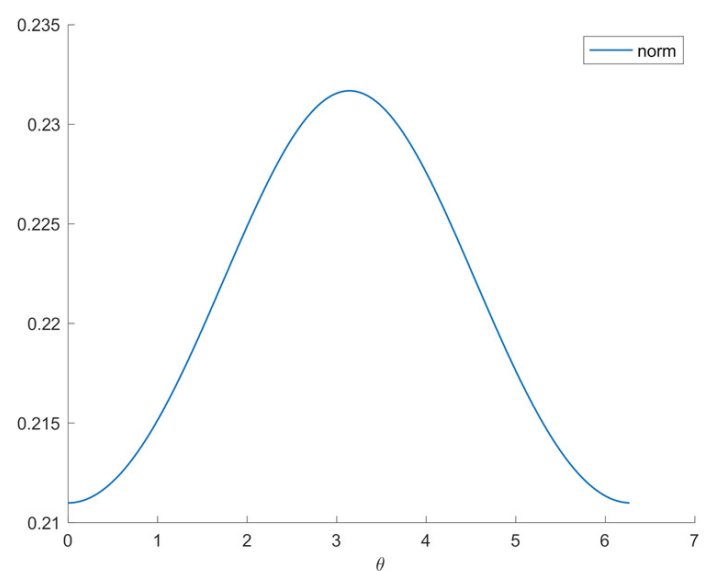

Figure 15. Value of $\sup _{\theta \in[0,2 \pi)}\left\|\left(e^{i \theta} E_{0}-A_{0}\right)^{-1}\left(e^{i \theta} \widetilde{E}-\widetilde{A}\right)\right\|$ with $\theta$ in Example 7. 


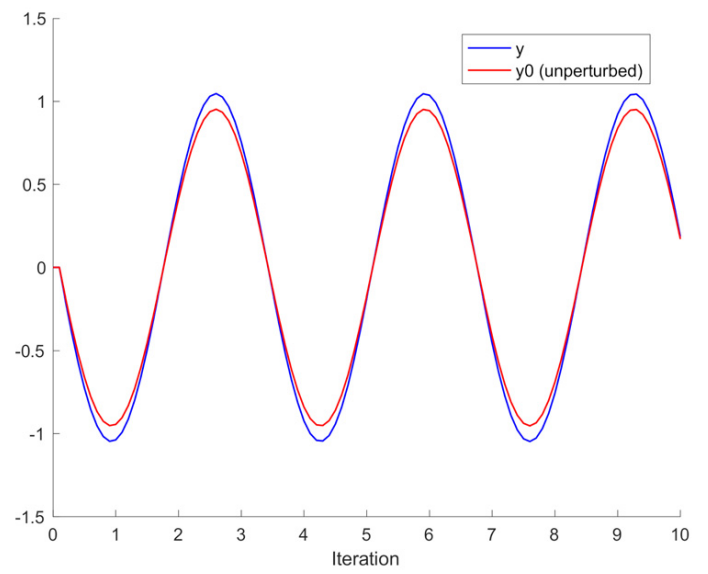

Figure 16. Output of the perturbed $(E, A)$ and unperturbed $\left(E_{0}, A_{0}\right)$ systems in Example 7.

\section{Conclusions}

This paper has described a general proposed singular regular dynamic discrete Leontief-type economic model which considers coupled economic production industries, recycling industries with used products, and non-renewable products. The model is subject to the usual driving demand plus a correcting control which is generated via feedback information of the economic production vector, recycling vector. and demand. The main objective is to study its reachability properties driven either by the demand vector or by the correcting control. It is well-known that reachability is a close concept to that of controllability of the state to the origin, but the main issue instead is to investigate the existence of a control which transfers any initial state to a prescribed final one in finite time. Due to the nature of the model (in particular, positive and non-singular regular), several specific difficulties have to be overcome, in particular, the achievement of the positivity of the solution sequence for any initial state under a positive control to be found which tracks a positive prefixed final state in finite time. Some constraints to be satisfied follow from the characteristics of the positive solution sequence and the positive control sequence which generates the solution. In particular, the final state is also subject to reinforced positivity constraints which depend on the initial conditions and the programmed final time for the solution to match the final prescribed state. It is assumed that the model is driven by both demand and a correcting control which can be, in turn, generated in the most general case by feedback information on the state variables and demand. The model is parameterized by the conventional input coefficients of production, the capital coefficients of production, the input coefficients of resources, showing the input of resources of industries which are required to produce a unit of product, the capital coefficients of recycling, used output production coefficients, recycling, as well as final consumption and the allocation matrix of output recycling coefficients related to the output of reused products. The meaning of the proposed feedback in the context of supply and demand regulation is also discussed with economic insight, in the sense that the supply is adjusted by the feedback-based correcting control using feedback information of the economic and re-usable goods and the demand. In the same way, the correcting control is the minus demand/supply error. Some related feedbacks ideas for more basic non-singular Leontief models and some of their extensions were commented in a nice way in [38]. It can also be pointed out that the accommodation of the supply to the demand is of practical interest in a number of economic models. See, for instance [39], where a contract-theoretic demand response is formulated via a maximization problem of the electricity market's utility. Several numerical examples related to the given theoretical framework have been discussed.

Author Contributions: Formal analysis, M.D.1.S.; supervision, S.A.-Q., A.I.; Software and programming, A.I.; writing original draft, M.D.I.S., S.A.-Q. and A.I.; funding acquisition, M.D.l.S.; investigation, M.D.I.S., S.A.-Q., A.I. All authors have read and agreed to the published version of the manuscript. 
Funding: RTI2018-094336-B-100 (MCIU/AEI/FEDER, UE) and Basque Government Grant IT1207-19.

Institutional Review Board Statement: No applicable.

Informed Consent Statement: No applicable.

Data Availability Statement: No applicable.

Acknowledgments: The authors are grateful to the Spanish Government for its support through grant RTI2018-094336-B-100 (MCIU/AEI/FEDER, UE) and to the Basque Government for its support through Grant IT1207-19.

Conflicts of Interest: The authors declare they have no conflict of interest.

\section{References}

1. Emmenegger, J.F.; Chable, D.; Nour Eldin, H.A.; Knolle, K. Sraffa and Leontief revisited, mathematical methods and models of a circular economy. In Monograph; de Gruyter-Oldenbourg, W., Ed.; De Gruyter: Berlin, Germany, 2020.

2. Feng, Y.; Yagoubi, M. Robust control of linear descriptor systems. In Studies in Systems, Decision and Control Series; No., 102; Kacprzyk, J., Ed.; Springer Nature: Singapore, 2017.

3. Banasiak, J.; Bobrowski, A.; Lachowicz, M.; Tomilov, Y. (Eds.) Semigroups of operators-theory and applications. In Springer Proceedings in Mathematics E Statistics; SOTA Kazimierz Dolny, Poland, Sept./Oct. 2018, In honour of J. Kisynski's 85 birthday; Springer Nature: Cham, Switzerland, 2020; Volume 325.

4. Soderholm, P. Environmental regulations and interfuel substitution in the power sector: A generalized Leontief model. Energy Environ. 2000, 11, 1-23. [CrossRef]

5. Dobos, I.; Floriska, A. A dynamic Leontief model with non-renewable resources. Econ. Syst. Res. 2005, 17, 317-326. [CrossRef]

6. Dobos, I.; Floriska, A. The resource conservation effect of recycling in a dynamic Leontief model. Int. J. Prod. Econ. 2007, 108, 334-340. [CrossRef]

7. Pavlova, N.G. Study of the continuous-time open dynamic Leontief model as a linear dynamical control system. Differ. Equ. 2019, 55, 111-116. [CrossRef]

8. Luenberger, D.G.; Arbel, A. Notes and comments: Singular dynamic Leontief systems. Econometrica 1977, 45, 991-995. [CrossRef]

9. Silva, M.S.; de Lima, T.P. Looking for negative solutions of a Leontief dynamical model. Linear Algebra Its Appl. 2003, 364, 291-306. [CrossRef]

10. Keller, A.V. On the computational efficiency of the algorithm of the numerical solution of optimal control problems for models of Leontieff type. J. Comp. Eng. Math. 2015, 2, 39-59. [CrossRef]

11. Shestakov, A.L.; Keller, A.V.; Sviridyuk, G.A. The theory of optimal measurements. J. Comp. Eng. Math. $2014,1,3-16$.

12. Shestakov, A.L.; Sviridyuk, G.A.; Butakova, M.D. The mathematical modelling of the production of construction mixtures with prescribed properties. Vestn. YuUrGU. Ser. Mat. Model. Progr. 2015, 8, 100-110. [CrossRef]

13. Levia, H.; Ríos-Bolivar, A.; Tineo-Moya, A.; Narváez, M. Análisis de la controlabilidad de sistemas descriptores semilineales. Cienc. Ing. 2017, 38, 187-196.

14. Faramondi, L.; Oliva, G.; Piemonte, V. Novel vulnerability metrics for interdependent systems based on system controllability. J. Phys. Conf. Ser. 2018, 1026, 1-10. [CrossRef]

15. Sugishita, K.; Asakura, Y. Vulnerability studies in the fields of transportation and complex networks: A citation network analysis. Public Transp. 2021, 13, 1-34. [CrossRef]

16. De Mesnard, L. Price consistency in the Leontief model? Cah. d'économie Polit. 2016, 71, 181-201. [CrossRef]

17. De Mesnard, L. Is the Ghosh model interesting? J. Reg. Sci. 2009, 49, 361-372. [CrossRef]

18. Sancho, F. An Armington-Leontief model. J. Econ. Struct. 2019, 8, 1-10. [CrossRef]

19. Kaczorek, T. Positive 1D and 2D systems. In Communications and Control Engineering Series; Sontag, E.D., Thoma, M., Eds.; Springer: Berlin, Germany, 2002

20. Conte, G.; Perdon, A.M.; Zattoni, E. Model matching problems for positive systems. IFAC Pap. 2020, 53, 4648-4653. [CrossRef]

21. Kaczorek, T.; Ruszewiski, R. Global stability of discrete-time nonlinear systems with descriptor standard and fractional positive linear parts and standard feedbacks. Arch. Control. Sci. 2020, 30, 667-681.

22. de la Sen, M.; Alonso-Quesada, S. A Control Theory point of view on Beverton-Holt equation in population dynamics and some of its generalizations. Appl. Math. Comput. 2008, 199, 464-481. [CrossRef]

23. de la Sen, M.; Alonso-Quesada, S. Model-matching-based control of the beverton-holt equation in ecology. Discret. Dyn. Nat. Soc. 2008, 2008, 793512. [CrossRef]

24. Krokavec, D.; Filasova, A. On control of discrete-time LTI positive systems. Appl. Math. Sci. 2017, 11, 2459-2476. [CrossRef]

25. Xu, S.; Lam, J. Robust stability for uncertain discrete singular systems with delay. Asian J. Control. 2003, 5, 399-405. [CrossRef]

26. Rami, M.A. Solvability of static output-feedback stabilization for LTI positive systems. Syst. Control. Lett. 2011, 60, 704-708. [CrossRef]

27. Steinberg, S.L.; Zingano, J.P.; Zingano, P.R. On nilpotent singular systems. J. Comput. Appl. Math. 2001, 137, 97-107. [CrossRef] 
28. Duan, G.R. Analysis and design of descriptor linear systems. In Advances in Mechanics and Mathematics; Gao, D., Ratiu, T., Eds.; Springer: New York, NY, USA, 2010; Volume 23.

29. De la Sen, M. On positivity of singular regular linear time-delay time-invariant systems subject to multiple internal and external incommensurate point delays. Appl. Math. Comput. 2007, 190, 382-401. [CrossRef]

30. de la Sen, M.; Alonso-Quesada, S. On the properties of reachability, observability, controllability and constructability of discretetime positive time-invariant linear systems with aperiodic choice of the sampling instants. Discret. Dyn. Nat. Soc. 2007, 2007, 1-23. [CrossRef]

31. Barnett, S. Matrices in Control Theory; Krieger Publishing: Malabar, FL, USA, 1984.

32. Moysis, L.; Mishra, V.K. Existence of reachable and observable triples of linear discrete-time descriptor systems. Circuits Syst. Signal Process. 2019, 38, 1086-1098. [CrossRef]

33. Kuo, I.-W. A note of factorizations of symmetric M-matrices. Linear Algebra Its Appl. 1977, 16, 217-220. [CrossRef]

34. Jacobson, D.H. Factorizations of singular M-matrices. Linear Algebra Its Appl. 1974, 9, 275-278. [CrossRef]

35. Franklin, G.; Powell, J.D. Digital Control of Dynamic Systems; Addison-Wesley Publishing Company: Reading, MA, USA, 1980.

36. Mertzios, B.G.; Lewis, F.L. Fundamental matrix of discrete singular systems. Circuits Syst. Signal Process. 1989, 8, 341-355. [CrossRef]

37. Rami, M.A.; Napp, D. Positivity of discrete singular systems and their stability: An LP- based approach. Automatica 2014, 50, 84-91. [CrossRef]

38. Hernández-Iglesias, C. Modelos dinámicos inter-sectoriales. Reflexiones críticas desde la Teoría de Control. Cuad. de Economía Span. J. Econ. Financ. 1983, 11, 249-264.

39. Irtija, N.; Sangoleye, F.; Tsiropoulou, E.E. Contract-theoretic demand response management in smart grid systems. IEEE Access 2020, 8, 184976-184987. [CrossRef] 